\title{
Discrete regulation of $\beta$-catenin-mediated transcription governs identity of intestinal epithelial stem cells
}

Costanza Borrelli ${ }^{1,2,4}$, Tomas Valenta ${ }^{1,3,4,{ }^{\star}, \text { Kristina Handler }}{ }^{2}$, Karelia Vélez ${ }^{2}$, Giulia Moro ${ }^{1}$, Atefeh Lafzi ${ }^{2}$, Laura de Vargas Roditi², George Hausmann ${ }^{1}$, Andreas E Moor ${ }^{2}$, Konrad Basler $^{1, *}$

1) Department of Molecular Life Sciences, University of Zurich, Winterthurerstrasse. 190, $\mathrm{CH}-8057$ Zurich, Switzerland

2) Institute of Molecular Cancer Research, University of Zurich, Winterthurerstrasse. 190, $\mathrm{CH}-8057$ Zurich, Switzerland

3) Institute of Molecular Genetics of the ASCR, v. v. i., Vídeňská 108314220 Prague 4, Czech Republic

4) These authors contributed equally

*Correspondence: konrad.basler@imls.uzh.ch, tomas.valenta@imls.uzh.ch 


\section{Abstract}

The homeostasis of the gut epithelium relies upon continuous renewal and proliferation of crypt-resident intestinal epithelial stem cells (IESCs). Wnt/ $\beta$-catenin signaling is required for IESC maintenance, however, it remains unclear how this pathway selectively governs the identity and proliferative decisions of IESCs. Here, we demonstrate that C-terminally-recruited transcriptional co-factors of $\beta$-catenin act as all-or-nothing regulators of Wnt-target gene expression. Blocking their interactions with $\beta$-catenin rapidly induces loss of IESCs and intestinal homeostasis. Conversely, N-terminally recruited co-factors fine-tune $\beta$-catenin's transcriptional output to ensure proper self-renewal and proliferative behaviour of IESCs. Impairment of N-terminal interactions triggers transient hyperproliferation of IESCs, eventually resulting in exhaustion of the self-renewing stem cell pool. IESC mis-differentiation, accompanied by intrinsic and extrinsic stress signalling results in a process resembling aberrant "villisation" of intestinal crypts. Our data suggest that IESC-specific Wnt/ $\beta$-catenin output requires discrete regulation of transcription by transcriptional co-factors. 


\section{Introduction}

Intestinal epithelial stem cells (IESCs) fuel the perpetual replenishment of the epithelial monolayer that lines the gut. They divide on average once a day to give rise to fast cycling progenitors (transit amplifiers, TA), which undergo several rounds of cell division before differentiating into cells of the absorptive (enterocytes) and the secretory lineage (Paneth, goblet, tuft and enteroendocrine cells $)^{1}$. Wnt/ $\beta$-catenin signalling is required for the maintenance of the intestinal epithelial stem cell pool ${ }^{2}$. However, to date, the gene regulatory networks governing the behaviour of IESCs remain elusive. The activity level of the Wnt pathway alone cannot explain the cell-type specific proliferative rate and self-renewal ability of IESCs, TAs and Paneth cells ${ }^{3,4}$. Hence, discrete regulation of Wnt-target gene expression may determine the identity of the self-renewing pool of IESCs.

A common extracellular signal may be translated into distinct cellular responses, and fate decisions, via the expression of specific cell-autonomous effectors. In the context of Wnt signalling, transcriptional co-factors that interact with $\beta$-catenin have been repeatedly implicated in tissue- and cell type-specific modulation of Wnt responses. C-terminal $\beta$-catenin interactors, such as CREB-binding protein (CBP) and p300, as well as members of the Mediator and of the RNA polymerase II-associated factor 1 (PAF1) complexes, are promiscuous co-activators that interact, besides with $\beta$-catenin, with several transcription factors $^{5,6}$. Conversely, B-cell lymphoma 9 (BCL9) or the paralog BCL9-like (BCL9L) specifically bind an $\mathrm{N}$-terminal moiety of $\beta$-catenin, and act as tissue-specific transcriptional effectors $^{7-11}$. While dispensable for normal intestinal homeostasis, BCL9/9L are required for intestinal regeneration upon insults ${ }^{12-15}$, suggesting that they might be necessary for the reconstitution of the stem cell pool. Moreover, compelling evidence has been provided that BCL9/9L plays a crucial role in maintaining tumor stemness in several murine models of colorectal cancer ${ }^{12-15}$.

It remains untested whether distinct regulation of Wnt-signaling outputs is connected to IESCspecific fate determination and self-renewal ability. In this study, we dissect the individual contributions of the $\mathrm{C}$ - and $\mathrm{N}$-terminal transcriptional branches of $\beta$-catenin to the maintenance of intestinal homeostasis. Using mutant $\beta$-catenin alleles that specifically impair the recruitment of $\mathrm{N}$ - or $\mathrm{C}$-terminal $\beta$-catenin's co-factors ${ }^{7}$, we show how discrete regulation of Wnt/B-catenin signalling does governs IESC identity and fate. While C-terminal co-actors act as an all-or-nothing switch for Wnt-target gene expression, $\mathrm{N}$-terminal co-activators are responsible for selective transcriptional modulation ensuring proper proliferation and selfrenewal of IESCs. 


\section{Results}

\section{Attenuation of $\mathrm{N}$ - vs. C-terminal $\beta$-catenin transcriptional outputs has contrasting effects on intestinal homeostasis}

We previously generated transgenic $\beta$-catenin (Ctnnb1) alleles harboring mutations that prevent interactions with $\mathrm{N}$ - or C-terminal transcriptional co-factors (NTFs and CTFs, respectively $)^{7}$. The D164A mutation abrogates interaction with NTFs, the $\Delta C$ truncation abrogates the interaction with the CTFs (Fig. 1a). To overcome embryonic lethality of these alleles, we used compound heterozygous mice carrying one mutant and one conditional $\beta$ catenin allele (Extended Data Fig. 1a). Similarly to constitutively hemizygous Ctnnb $1^{\text {KO/wt }}$ mice, Ctnnb1 $1^{D 164 A / f l o x}$ and Ctnnb1 $1^{\Delta C / f l o x}$ animals show no overt abnormalities, indicating haplosufficiency of $\beta$-catenin for the maintenance of intestinal homeostasis. Combination with the villin-CreER ${ }^{T 2}$ driver ${ }^{16}$ enables the inducible deletion of the conditional $\beta$-catenin allele $\left(C t n n b 1^{f l o x}\right.$ ) specifically in the intestinal epithelium, thus leaving the Wnt-transcriptional outputs solely under the control of mutant $\beta$-catenin (D164A or $\Delta \mathrm{C}$ ). While villin-CreER ${ }^{T 2}$;Ctnnb ${ }^{\text {wtfflox }}$ (control) mice are perfectly viable and indistinguishable from homozygous wild type animals, the presence of only mutant $\beta$-catenin is lethal: villin-CreER ${ }^{T 2} ; C \operatorname{Ctnnb} 1^{\Delta C / f l o x}(\Delta \mathrm{C})$ animals exhibit atrophic crypts at $4 \mathrm{~d}$ post Cre induction (pi) (Extended Data Fig. 1b). This is in accordance with our previous results in villin-CreER ${ }^{T 2}$;Ctnnb $1^{\text {dm/flox }}$ animals, which express double mutant (dm) $\beta$-catenin harboring both $\mathrm{N}$ - and $\mathrm{C}$-terminal mutations ${ }^{17}$. Unlike $\mathrm{dm}$ and $\Delta \mathrm{C}$ animals, villinCreER ${ }^{T 2}$; Ctnnb1 $^{\text {D164A/flox }}(\mathrm{D} 164 \mathrm{~A})$ animals only reach humane endpoint at $7 \mathrm{~d}$ pi, as they suffer from severe colitis (Extended Data Fig. 1c). The different intestinal phenotypes that arise upon blocking $\mathrm{C}$ - or $\mathrm{N}$-terminal transcriptional outputs of $\beta$-catenin are consistent with distinct phenotypic impacts of these mutants during embryonic development ${ }^{7}$.

Consistent with a fast turnover of intestinal epithelial cells, we observed depletion of wt (flox) $\beta$-catenin $2 d$ pi, while the integrity of intestinal crypts remained unaltered (Extended Data Fig. $1 \mathrm{~d}, \mathrm{e})$. We performed RNA sequencing of bulk preparations of small intestinal epithelium $2 \mathrm{~d}$ pi from control, $\triangle \mathrm{C}, \mathrm{D} 164 \mathrm{~A}, \mathrm{dm}$ and $\mathrm{KO}$ animals. Principal component analysis indicated surprising differences of impairing $\mathrm{N}$ - versus $\mathrm{C}$-terminal interactions on the epithelial transcriptome (Fig. 1b). The differentially expressed genes (DEGs, logFC > $2 \mid, p<0.05$ ) of $\triangle \mathrm{C}$ animals broadly overlapped with those of $\mathrm{dm}$ and full knock-out $(\mathrm{KO})$ animals, indicating that $\mathrm{C}$-terminally mutated $\beta$-catenin is not able to sustain Wnt signalling levels required for proper renewal of the intestinal epithelium (Fig. 1c). Attenuating C-terminal $\beta$-catenin transcriptional outputs in the intestinal epithelium caused a loss of IESCs, and an attrition of proliferation in the transient amplifier compartment, as evidenced by IESC gene and proliferative marker expression (Fig. 1d), gene set enrichment analysis (GSEA, Extended Data Fig. 1f $)^{18}$, as well as protein stainings and RNA in situ hybridization (Fig. 1e-g). Notably, as previously reported for the $\beta$-catenin-dm animals ${ }^{7,17}$, the observed phenotype in $\Delta \mathrm{C}$ animals is entirely connected to transcriptional outputs, and not attributable to loss of epithelial adhesiveness, as in the case of complete $\beta$-catenin loss. Contrary to what was observed in $\Delta \mathrm{C}$ mice, the transcriptomic changes induced in $\beta$-catenin-D164A animals (i.e. $\mathrm{N}$-terminal mutant) only minimally overlapped with those induced by the loss of $\beta$-catenin (Fig. 1c). The exclusivity of DEGs in D164A-mutant can be partially attributed to a D164A-specific enrichment of genes expressed by infiltrating immune cells (Extended Data Fig. 1g). As opposed to what we observed in $\triangle \mathrm{C}$ crypts, the expression of Wnt targets, IESC genes and proliferation markers, was significantly increased in D164A crypts 2d pi (Fig. 1e-f). Moreover, D164A mutant crypts displayed a significant increase of the Lgr5+ area, indicating an 
expansion of the IESC compartment (Fig. 1g, Extended Data 1h). Furthermore, the presence of Ki67+ cells at the bottom of D164A crypts indicates increased IESC proliferation (Fig. 1e,f). Consistent with hyperproliferation in the stem cell compartment, Myc expression was upregulated in the bottom of the crypt, as shown by single molecule fluorescent in situ hybridization (smFISH) (Fig. 1h). These results indicate that attenuating $\beta$-catenin's $\mathrm{N}$-terminal transcriptional outputs increased proliferation of IESCs and resulted in an expansion of the stem cell compartment. On the contrary, preventing interactions to CTFs completely represses the Wnt-outputs, including proliferation and IESC-associated genes, hence compromising stem cell maintenance.

\section{Intestinal crypts lacking the output via of $\mathbf{N}$-terminal recruited co-factors exhibit stem cell loss and secretory hyperplasia $4 \mathrm{~d}$ pi}

Unexpectedly, in contrast to $2 \mathrm{~d}$ pi, expression of Wnt-target genes, as well as IESC genes and proliferative markers was completely abrogated in D164A crypts 4d pi (Fig. 2a). Moreover, crypt morphology was profoundly altered by the appearance of large granular cells that express the secretory lineage marker Sox9 (Fig. 2a) and are double positive for lysozyme (Lyz) and mucins (stained by alcian blue, AB) (Fig. 2a,b). These so-called intermediate cells, which share both Paneth (lysozyme) and goblet cell traits (mucins), are the result of stem cell mis-differentiation, and have been observed upon experimental perturbation of Notch, Wnt or EGF signalling in the intestinal epithelium ${ }^{19-23}$. However, the molecular mechanisms underlying their appearance in the tissue is largely unknown. Interestingly, a shift towards secretory lineage differentiation was observed upon deletion of $\mathrm{N}$-terminal co-factors BCL9/9L in $\mathrm{APC}^{\mathrm{min}}$ tumors ${ }^{15}$, suggesting that the NTFs might repress specification of the secretory lineage.

Taken together, these results indicate that neither $\mathrm{C}$ - nor $\mathrm{N}$-terminally mutated $\beta$-catenin is haplosufficient in the intestinal epithelium, as these mutants are not able to substitute for the wild type allele. However the cellular dynamics leading to crypt collapse are profoundly distinct (Fig. 2c). Similar to the complete deletion of $\beta$-catenin, blocking its CTF recruitment rapidly caused a lethal loss of stem cells. On the other hand, impairing interaction with BCL9/9L triggered transient hyperproliferation of IESCs, followed by their aberrant differentiation.

\section{Hyperproliferative IESCs expressing only Ctnnb1 ${ }^{D 164 A}$ have an increased ability to form organoids}

Recent work indicated that intestinal organoids could not be established from BCL9/9Ldeficient crypts isolated $4 \mathrm{~d} \mathrm{pi}^{14}$. However, we hypothesized that expansion of the proliferative IESC compartment in D164A crypts observed 2d pi would translate into increased organoid formation rate. Thus, we devised an organoid formation assay to test this (Fig. 3a): we isolated intestinal crypts from villin-CreER ${ }^{T 2} ;$ Ctnnb $^{\text {wtfllox }}$ (control) and villin-CreER ${ }^{T 2}$;Ctnnb ${ }^{\text {D164Aflox }}$ (D164A) animals 0, 2 and 4d post Cre induction, seeded equal amounts in Matrigel and quantified the number of organoids formed $7 \mathrm{~d}$ post seeding. Intriguingly, hemizygous control crypts showed a drastic reduction in organoid formation rate upon loss of the conditional allele (Fig. 3b). Indeed, in contrast to the in vivo situation, we found that $\beta$-catenin is not haplosufficient in vitro: neither crypts isolated from constitutively hemizygous mice, nor upon acute Cre induction (in vivo and in vitro) can be propagated as intestinal organoids (Fig. 3c and Extended Data Fig. 2a). Moreover, crypts isolated from Ctnnb1 $1^{\Delta C / f l o x}$ and Ctnnb1 $1^{\text {dm/flox }}$ animals also fail to grow in vitro (Extended Data Fig. 2b, indicating that two copies of Cterminally transcriptionally active $\beta$-catenin alleles are necessary to maintain Wnt-target gene expression required by intestinal organoids. The differential $W n t / \beta$-catenin-signalling 
requirements of intestinal crypts in vitro might be due to the fact that organoids lack a mesenchymal support niche, and that they closely resemble a regenerative, rather than homeostatic state of intestinal crypts. Nonetheless, D164A crypts isolated $2 \mathrm{~d}$ pi exhibited a 1.6-fold increase in organoid formation rate compared to non-induced (Ctnnb $1^{\text {D164A/flox }}$ ) crypts, and a 10-fold increase compared to the respective control (Fig. 3c). Morphologically, organoids derived from hyperproliferative crypts showed increased cell proliferation and budding (Fig. 3c). Consistent with the transient nature of the hyperproliferation observed in vivo, these organoid lines obtained from D164A crypts $2 \mathrm{~d}$ pi cannot be maintained in culture, and died upon first passaging (post splitting panels, Fig. 3c). Crypts isolated from D164A animals $4 \mathrm{~d}$ pi failed to establish organoids in vitro, in agreement with the loss of stem cell and proliferation markers observed in the tissue (Fig. 3b). In sum, these results in vitro recapitulate and functionally underscore our observations in vivo.

\section{Myc-E2F-driven proliferation leads to exhaustion of the stem cell pool in N-terminal mutant (D164A) animals}

To dissect the gene regulatory networks underlying the transient hyperproliferation of IESCs triggered by impaired $\beta$-catenin-NTF interactions, we performed droplet-based single cell RNA sequencing (scRNAseq) of small intestinal crypts isolated from control and D164A intestines at 0,2 and $4 \mathrm{~d}$ pi. We focused our analysis on the gene expression changes occurring over time in stem cells and early progenitors (SCEP cells), which we annotated manually based on marker gene expression (Extended Data Fig. 3a-c). To disentangle the transcriptomic effects caused by impaired $\mathrm{N}$-terminal interactions from those arising due to the acute loss of the conditional $\beta$-catenin allele, we devised a normalization procedure using the control time series data (detailas in Methods).

Confirming the data presented above (Fig. 1d-g), D164A SCEP cells exhibited transient upregulation of IESC and proliferation markers $2 \mathrm{~d}$ pi, followed by increased expression of differentiation markers $4 d$ pi (Extended Data Fig. $3 d$ ). This is highlighted by diffusion maps ${ }^{24}$ as a shift along the stem cell differentiation continuum: SCEP cells isolated $2 \mathrm{~d}$ pi have a significantly more "stem-like" distribution, whilst SCEP cells from D164A crypts 4d pi are more shifted towards differentiation (Fig. 4a,b). The upregulation of stem cell markers $2 \mathrm{~d}$ pi was accompanied by a significant increase in the mean expression levels of cell cycle-related genes $^{25}$ (Fig. 4c). Supervised pseudotime analysis ${ }^{26}$ confirmed that stem cell and proliferation markers exhibit a similar expression profile over time, increasing $2 \mathrm{~d} p \mathrm{p}$, and subsequently dropping 4d pi (Fig. 4d). We had observed increased Myc expression in D164A crypts 2d pi (Fig. 1h), we therefore asked if Myc-driven proliferation was causing increased cell cycle rate of SCEP cells. At $2 \mathrm{~d}$ pi, Myc-dependent Wnt-targets were significantly enriched among DEGs between 0d and 2d pi (MSigDB name: M1757, normalized enrichment score (NES)=1.68, $\mathrm{p}<0.05$, from ${ }^{27}$ ) (labeled as APC-targets in Fig. 4e). Moreover, the signature of E2F - the major cell cycle regulator, and a Myc-target - was significantly enriched (M5901, NES=1.56, $p<0.05$ ). Also enriched were genes involved in cell cycle $(\mathrm{M} 14460, \mathrm{NES}=1.65, p<0.05)$ and $\mathrm{G} 2 \mathrm{M}$ checkpoint (M5901, NES=1.72, $\mathrm{p}<0.05)$ (Fig. 4d). These proliferation signatures were all significantly depleted $4 d$ pi (NES<-1, p<0.05), reflecting abrupt proliferative arrest (Fig. 4c,d). We wondered whether the observed Myc-E2F-induced proliferation burst induced an en-bloc conversion of D164A IESCs to TAs, which are known to exhibit higher expression of proliferative $\mathrm{Wnt}$ targets ${ }^{28}$. To test this hypothesis, we trained a logistic regression model on TAs and IESCs obtained from a publicly available single cell dataset of murine small intestinal epithelium ${ }^{29}$, and tested SCEP cells from $0 \mathrm{~d}$ and $2 \mathrm{~d}$ pi against this model. Comparison of the distribution of predicted model responses from the two timepoints shows a significant shift of 
$2 \mathrm{~d}$ pi cells towards TA traits (Extended Data Fig. 3d). Cumulatively, our results indicate that impairing $\beta$-catenin-NTF interactions transiently activates an Myc-E2F-driven proliferation program in IESCs, which results in exhaustion of the self-renewing IESC pool.

\section{Environmental and cell-intrinsic stress signals induce mis-differentiation}

Gene set enrichment analysis (GSEA) of time-varying genes in SCEP cells also revealed an increasing extrinsic and intrinsic stress signaling in N-terminally mutated (D164A) crypts. Signatures of TNFa signalling and interferon- $y$ signalling are significantly upregulated in D164A SCEP cells (M5913, NES=2.32, $p<0.001$ and M5890, NES $=2.08, p<0.001$, respectively). Accordingly, the cytokine-induced genes Irf7, Fos, Jun, and Junb are among the most upregulated transcription factors according to our supervised pseudotime analysis (Extended Data Fig. 3f). Moreover, we found genes involved in endogenous antigen presentation to be significantly upregulated over time in D164A SCEP cells (M16750, NES=3.01, $p<0.001)$. Interestingly, a proliferative subset of IESCs has recently been described to function as unconventional antigen presenting cells, orchestrating interactions with immune cells in the intestine via expression of MHC class II complexes ${ }^{30}$. In line with these results, and together with the lymphocyte recruitment indicated by the RNASeq data (Extended Data Fig. 1g), we observed infiltration of CD45+ cell in the pericryptic zone, as well as within crypts, of D164A animals 4d pi (Fig. 4f).

The unfolded protein response (UPR) is a major cell-intrinsic stress response. Key mediators of the UPR - Xbp1, Creb3, and Creb3/3 - were among the upregulated transcription factors over time (Extended Data Fig. 3f). Creb3/3 is normally a strongly zonated gene; its expression increasing along the crypt-villus axis, with peak expression at the villus tip ${ }^{31}$. We confirmed its ectopic expression in D164A crypts 4d pi using smFISH (Extended Data Fig. 3g). Consistent with an UPR, genes involved in translation initiation and ribosomal function are significantly downregulated in D164A crypts (respectively M27686, NES=-2.52, $p<0.05$ and M189, NES=3.26, p<0.001) (Fig. 4e). Interestingly, both UPR ${ }^{32}$ and interferon- $y$ signalling ${ }^{30}$ have been shown to induce differentiation of IESCs. Furthermore, antigen presentation is intrinsically linked to UPR ${ }^{33}$. For instance, prior to the discovery of its role in UPR, Xbp1 was originally discovered as a regulator of class II major histocompatibility genes ${ }^{34,35}$. Altogether, these results suggest that, following hyperproliferation of IESCs, a combination of environmental (cytokines) and cell-intrinsic stress induces loss of stem cells and secretory hyperplasia of D164A crypts, resulting in loss of stem cells and subsequently failure of epithelial homeostasis.

\section{The chromatin landscape of D164A crypts reflects the transition to proliferative arrest and mis-differentiation}

Our analyses revealed rapid and profound transcriptomic and morphological changes in the crypts upon impairment of $\beta$-catenin-NTF interactions (D164A mutant). We reasoned that profiling the chromatin landscape of D164A crypts would provide insight into the gene regulatory mechanisms responsible for such drastic reprogramming of epithelial cells. To this end, we performed Assay for Transposase-Accessible Chromatin using sequencing (ATACSeq) of control and D164A crypts isolated $2 \mathrm{~d}$ pi. Differential peak analysis revealed that 1469 ATAC-peaks are differentially accessible (logFC $>|1| \& p<0.01$ ) in D164A crypts, compared to control crypts (Fig. 5a,b). We subjected these peaks to motif analysis with HOMER $^{36}$, and found a significant enrichment (Benjamini-corrected q-value $<0.001$ ) of motifs associated with transcription factors involved in intestinal differentiation, such as hepatocyte nuclear factors (HNF4a,1,1b), ETS-related transcription factors (EHF, ELF3,5) Caudal Type Homeobox (Cdx2,4), GATA-binding factors and Krüppel-like factors (Extended Data Fig. 4a). 
These findings correlate with increased expression of secretory and absorptive lineage markers in D164A crypts seen at 4d pi. Moreover, peaks annotated to Wnt target genes (Tcf7/1/2, Axin2, Fzd7, Sp5), Wnt-inhibitors (Tle3, Trabd2b, Wif1, Dab2ip, Amer3) and cell cycle genes (E2f1, Cenpf, Cebpe) Fig. among the lost peaks (Fig. 5b). These results indicate that the chromatin landscape $2 \mathrm{~d}$ pi preludes the proliferative arrest and secretory hyperplasia observed in N-terminal mutants (D164A) 4d pi. Indeed, we found that a significant fraction of pathways overrepresented in D164A vs control crypts according to our ATAC-peak analysis (2d pi) were also overrepresented in the transcriptome of D164A mutant crypts $4 \mathrm{~d}$ pi (Extended Data Fig. 4b) .

\section{JNK signalling mediates major chromatin remodelling in D164A crypts}

In line with our scRNASeq results, in the ATACSeq data we found a strong enrichment of motifs bound by transcription factors downstream of the c-Jun N-terminal kinases (JNK) signalling, namely the AP-1 transcription factors Fra1/2, Fos, Fosl2, JunB, Atf3 and BATF (Fig. 5c). Cumulatively, these motifs were enriched in 220 out of the 600 gained peaks in D164A (37\%), including peaks mapped to Fos, as well as Mapk8 (JNK) and the closely related Mapk13 (SAPK4), indicating robust JNK-mediated activation of gene expression (Fig. $5 \mathrm{~d}$ and Extended Data Fig. 4a). We validated JNK pathway activation by immunofluorescence, which reveals strong nuclear accumulation of phosphorylated JNK (pJNK) in D164A, but not in control crypts 4d pi (Fig. 5e). Similar to Creb3/3, the expression of transcription factors Fos and JunB is normally restricted to enterocytes at the villus tip ${ }^{31,37}$. However, in D164A animals $4 \mathrm{~d}$ pi, Fos and JunB transcripts are ectopically expressed in crypts (Fig. 5f), corroborating the ATAC-Seq and scRNASeq results. Moreover, the transmembrane mucin glycoprotein Muc13, which was independently shown to be induced by JNK activity ${ }^{38}$, also Fig. among the gained peaks in D164A mutant crypts (Fig. 5b), in line with increased alcian blue positivity of hyperplastic and mis-differentiated intermediate cells $4 \mathrm{~d}$ pi. Altogether, these results indicate that, following lack of $\beta$-catenin-NTF interactions, JNK signalling is activated. While misdifferentiation of IESCs upon perturbation of niche signalling has been previously described, this is, to our knowledge, the first time the JNK pathway has been implicated in loss of intestinal epithelial stem cells. 


\section{Discussion}

We have previously shown that the $\mathrm{C}$ - and $\mathrm{N}$-terminal transcriptional outputs of Wnt/ $\beta$-catenin signalling have distinct and independent functions during embryonic development ${ }^{7}$. In this study, we aimed to uncover their specific contributions to the maintenance of adult intestinal epithelial stem cell homeostasis. Our data strongly suggest that C-terminally recruited coactivators act as a binary on-off-switch, and are therefore essential for the basal $\beta$-cateninmediated transcription of Wnt-target genes. Conversely, N-terminal co-factors fine-tune $\beta$ catenin's transcriptional output to the levels required for proper proliferation and self-renewal of IESCs. This selective transcriptional modulation may be key in preserving "just-right" levels of Wnt signaling, which have been implicated in IESC homeostasis both physiological and in tumor context ${ }^{39}$.

While increasing attention has been devoted to the role of BCL9/9l in maintaining colorectal cancer stemness, the function of $\beta$-catenin-NTF-interactions in healthy intestinal homeostasis has been overlooked. This might be due to fact that BCL9/9L loss in the intestinal epithelium only transiently induces downregulation of IESC markers ${ }^{12-15}$. However, compelling evidence has recently been provided that the intestinal IESC niche is readily regenerated upon perturbations, as differentiated cells can revert to stem cells ${ }^{40-43}$. Epithelial plasticity can thus mask the effects of loss of function studies of proteins, such as BCL9/9L, whose contribution to intestinal homeostasis only becomes apparent when the epithelial stem cell niche is challenged. Our data suggest that $\mathrm{N}$-terminal co-factors selectively restrain $\mathrm{Wnt} / \mathrm{\beta}$-catenin outputs that promote Myc-E2F-driven proliferation, thereby ensuring the maintenance of a selfrenewing pool of IESCs. Indeed, in N-terminal mutant (D164A) crypts, excessive proliferation of IESCs rapidly led to exhaustion of the stem cell pool. Concomitantly with robust JNK pathway activation, the crypts underwent a profound mis-differentiation and "villisation". We show ectopic crypt expression of gene programs characteristic of enterocytes at the villus tip ${ }^{31,37}$, where terminally differentiated cells are eliminated by apoptosis. Mounting inflammation and colitis eventually resulted in a lethal loss of intestinal function. Further studies should dissect the role of immune cell-derived signals for maintenance of intestinal homeostasis in both physiological and perturbed conditions.

The cellular dynamics of crypt collapse in N-terminal mutant animals are in striking contrast with the rapid crypt atrophy observed upon $\beta$-catenin deletion, or lack of interactions with $\mathrm{C}$ terminally recruited co-factors. The results presented herein (Fig. 6) provide evidence that discrete regulation of $\beta$-catenin transcriptional outputs preserves the narrow window of Wntpathway activity and cellular specificity required to govern the identity and fate of intestinal epithelial stem cells. 


\section{Author Contributions}

CB designed and performed experiments, collected, analyzed and interpreted data and wrote the manuscript. TV initiated and conceived the research, designed and performed experiments, interpreted data and wrote the manuscript. $\mathrm{KH}, \mathrm{KV}$ and $\mathrm{GM}$ assisted with the experiments. $\mathrm{KH}, \mathrm{AL}$ and LVR helped with the bioinformatic analysis. $\mathrm{GH}$ and EAM discussed the data and assisted with manuscript preparation. EAM supported the research. KB initiated and supported the research, discussed the data and assisted with manuscript preparation.

\section{Acknowledgements}

S. Robine kindly shared the Villin-Cre ${ }^{E R T 2}$ strain with us. We are grateful to C. Cantù (Linköping University, Sweden) for valuable comments and to S. Janjuha and W. Macnair for advice. We appreciate discussions with members of the Basler and Moor lab, in particular with $\mathrm{N}$. Doumpas and J. Hilchenbach. For technical help we thank E. Escher and the Functional Genomics Center Zurich. This work was supported by the Swiss National Science Foundation, the Swiss Cancer League, the University of Zurich Research Priority Program (URPP) "Translational Cancer Research" and the Kanton of Zürich. TV is supported by Czech Science Foundation grant 18-21466S and is a fellow of the URPP Translational Cancer Research.

\section{Conflict of Interetst}

The authors declare no conflict of interets. 
bioRxiv preprint doi: https://doi.org/10.1101/2020.05.19.103499. this version posted May 20, 2020. The copyright holder for this preprint (which was not certified by peer review) is the author/funder. All rights reserved. No reuse allowed without permission.

\section{References}

1. Barker, N. et al. Identification of stem cells in small intestine and colon by marker gene Lgr5. Nature 449, 1003-1007 (2007).

2. Sato, T. et al. Paneth cells constitute the niche for Lgr5 stem cells in intestinal crypts. Nature 469, (2011).

3. Schepers, A. G., Vries, R., Van Den Born, M., Van De Wetering, M. \& Clevers, H. Lgr5 intestinal stem cells have high telomerase activity and randomly segregate their chromosomes. EMBO J. (2011). doi:10.1038/emboj.2011.26

4. Carroll, T. D., Newton, I. P., Chen, Y., Blow, J. J. \& Näthke, I. Lgr5+ intestinal stem cells reside in an unlicensed G1 phase. J. Cell Biol. (2018). doi:10.1083/jcb.201708023

5. Mosimann, C., Hausmann, G. \& Basler, K. $\beta$-Catenin hits chromatin: Regulation of Wnt target gene activation. Nat. Rev. Mol. Cell Biol. 10, 276-286 (2009).

6. Valenta, T., Hausmann, G. \& Basler, K. The many faces and functions of beta-catenin. EMBO J. 31, 2714-2736 (2012).

7. Valenta, T. et al. Probing transcription-specific outputs of $\beta$-catenin in vivo. Genes Dev. 25, 2631-2643 (2011).

8. Cantù, C. et al. The Pygo2-H3K4me2/3 interaction is dispensable for mouse development and Wnt signaling-dependent transcription. Development 140, 2377-2386 (2013).

9. Cantù, C. et al. Pax6-dependent, but $\beta$-catenin-independent, function of Bcl9 proteins in mouse lens development. Genes Dev. 28, 1879-1884 (2014).

10. Cantù, C. et al. A cytoplasmic role of Wnt/b-catenin transcriptional cofactors Bcl9, Bcl9l, and Pygopus in tooth enamel formation. Sci. Signal. 10, 1-11 (2017).

11. Cantù, C. et al. Mutations in Bcl9 and Pygo genes cause congenital heart defects by tissue-specific perturbation of Wnt/ $\beta$-catenin signaling. Genes Dev. 32, 1443-1458 (2018).

12. Deka, J. et al. Bcl9/Bcl9l are critical for Wnt-mediated regulation of stem cell traits in colon epithelium and adenocarcinomas. Cancer Res. 70, 6619-6628 (2010).

13. Moor, A. E. et al. BCL9/9L- $\beta$-catenin Signaling is Associated With Poor Outcome in Colorectal Cancer. EBioMedicine 2, 1932-1943 (2015).

14. Gay, D. M. et al. Loss of BCL9/9l suppresses Wnt driven tumourigenesis in models that recapitulate human cancer. Nat. Commun. 1-16 doi:10.1038/s41467-019-08586-3

15. Mieszczanek, J., Tienen, L. M. Van, Ibrahim, A. E. K., Winton, D. J. \& Bienz, M. Bcl9 and Pygo synergise downstream of Apc to effect intestinal neoplasia in FAP mouse models. Nat. Commun. doi:10.1038/s41467-018-08164-z

16. El Marjou, F. et al. Tissue-specific and inducible Cre-mediated recombination in the gut epithelium Genesis 39, 186-193 (2004).

17. Valenta, T. et al. Wnt Ligands Secreted by Subepithelial Mesenchymal Cells Are Essential for the Survival of Intestinal Stem Cells and Gut Homeostasis. Cell Rep. 15, 911-918 (2016).

18. Subramanian, A. et al. Gene set enrichment analysis: A knowledge-based approach for interpreting genome-wide expression profiles. Proc. Natl. Acad. Sci. U. S. A. (2005). doi:10.1073/pnas.0506580102

19. van Es, J. H. et al. A Critical Role for the Wnt Effector Tcf4 in Adult Intestinal Homeostatic Self-Renewal. Mol. Cell. Biol. (2012). doi:10.1128/mcb.06288-11

20. VanDussen, K. L. et al. Notch signaling modulates proliferation and differentiation of intestinal crypt base columnar stem cells. Development (2012). doi:10.1242/dev.070763

21. Tian, H. et al. Opposing Activities of Notch and Wnt Signaling Regulate Intestinal Stem Cells and Gut Homeostasis Report Opposing Activities of Notch and Wnt Signaling Regulate Intestinal Stem Cells and Gut Homeostasis. CellReports 11, 33-42 (2015).

22. Koppens, M. A. J. et al. Deletion of Polycomb Repressive Complex 2 From Mouse Intestine Causes Loss of Stem Cells. Gastroenterology (2016). doi:10.1053/j.gastro.2016.06.020

23. Chiacchiera, F. \& Pasini, D. Control of adult intestinal identity by the Polycomb repressive machinery. Cell Cycle (2017). doi:10.1080/15384101.2016.1252582

24. Haghverdi, L., Buettner, F. \& Theis, F. J. Diffusion maps for high-dimensional single-cell analysis of differentiation data. Bioinformatics (2015). doi:10.1093/bioinformatics/btv325

25. Kowalczyk, M. S. et al. Single-cell RNA-seq reveals changes in cell cycle and differentiation programs upon aging of hematopoietic stem cells. Genome Res. (2015). doi:10.1101/gr.192237.115 
bioRxiv preprint doi: https://doi.org/10.1101/2020.05.19.103499. this version posted May 20, 2020. The copyright holder for this preprint (which was not certified by peer review) is the author/funder. All rights reserved. No reuse allowed without permission.

26. Macnair, W. \& Claassen, M. psupertime: supervised pseudotime inference for single cell RNA-seq data with sequential labels. bioRxiv (2019). doi:10.1101/622001

27. Sansom, O. J. et al. Myc deletion rescues Apc deficiency in the small intestine. 446, 3-6 (2007).

28. Fevr, T., Robine, S., Louvard, D. \& Huelsken, J. Wnt/ $\beta$-Catenin Is Essential for Intestinal Homeostasis and Maintenance of Intestinal Stem Cells. Mol. Cell. Biol. 21, (2007).

29. Haber, A. L. et al. A single-cell survey of the small intestinal epithelium. Nature 551, 333-339 (2017).

30. Biton, M. et al. T Helper Cell Cytokines Modulate Intestinal Stem Cell Renewal and Differentiation. Cell (2018). doi:10.1016/j.cell.2018.10.008

31. Moor, A. E. et al. Spatial Reconstruction of Single Enterocytes Uncovers Broad Zonation along the Intestinal Villus Axis. Cell (2018). doi:10.1016/j.cell.2018.08.063

32. Heijmans, J. et al. ER Stress Causes Rapid Loss of Intestinal Epithelial Stemness through Activation of the Unfolded Protein Response. Cell Rep. (2013). doi:10.1016/j.celrep.2013.02.031

33. Osorio, F., Lambrecht, B. N. \& Janssens, S. Antigen presentation unfolded: identifying convergence points between the UPR and antigen presentation pathways. Current Opinion in Immunology (2018). doi:10.1016/j.coi.2018.04.020

34. Ono, S. J., Liou, H. C., Davidon, R., Strominger, J. L. \& Glimcher, L. H. Human X-box-binding protein 1 is required for the transcription of a subset of human class II major histocompatibility genes and forms a heterodimer with c-fos. Proc. Natl. Acad. Sci. U. S. A. (1991). doi:10.1073/pnas.88.10.4309

35. Iwakoshi, N. N., Lee, A. H. \& Glimcher, L. H. The X-box binding protein-1 transcription factor is required for plasma cell differentiation and the unfolded protein response. Immunological Reviews (2003). doi:10.1034/j.1600-065X.2003.00057.X

36. Heinz, S. et al. Simple Combinations of Lineage-Determining Transcription Factors Prime cis-Regulatory Elements Required for Macrophage and B Cell Identities. Mol. Cell (2010). doi:10.1016/j.molcel.2010.05.004

37. Halpern, K. B. et al. Lgr5+ telocytes are a signaling hub at the intestinal villus tip. bioRxiv (2019). doi:10.1101/850909

38. Chauhan, S. C. et al. Expression and functions of transmembrane mucin MUC13 in ovarian cancer. Cancer Res. (2009). doi:10.1158/0008-5472.CAN-08-0587

39. Albuquerque, $\mathrm{C}$. The 'just-right' signaling model: APC somatic mutations are selected based on a specific level of activation of the beta-catenin signaling cascade. Hum. Mol. Genet. (2002). doi:10.1093/hmg/11.13.1549

40. Bankaitis, E. D., Ha, A., Kuo, C. J. \& Magness, S. T. Reserve Stem Cells in Intestinal Homeostasis and Injury. Gastroenterology (2018). doi:10.1053/j.gastro.2018.08.016

41. Santos, A. J. M., Lo, Y. H., Mah, A. T. \& Kuo, C. J. The Intestinal Stem Cell Niche: Homeostasis and Adaptations. Trends in Cell Biology (2018). doi:10.1016/j.tcb.2018.08.001

42. Yousefi, M., Li, L. \& Lengner, C. J. Hierarchy and Plasticity in the Intestinal Stem Cell Compartment Trends in Cell Biology (2017). doi:10.1016/j.tcb.2017.06.006

43. de Sousa e Melo, F. \& de Sauvage, F. J. Cellular Plasticity in Intestinal Homeostasis and Disease. Cell Stem Cell (2019). doi:10.1016/j.stem.2018.11.019

44. Brault, V. et al. Inactivation of the beta-catenin gene by Wnt1-Cre-mediated deletion results in dramatic brain malformation and failure of craniofacial development. Development 128, 1253-1264 (2001).

45. Sato, T. et al. Growing Self-Organizing Mini-Guts from a Single Intestinal Stem Cell: Mechanism and Applications. Science (80-. ). 340, 241-260 (2013).

46. Lyubimova, A. et al. Single-molecule mRNA detection and counting in mammalian tissue. Nat. Protoc. (2013). doi:10.1038/nprot.2013.109

47. Itzkovitz, S. et al. Single-molecule transcript counting of stem-cell markers in the mouse intestine. Nat. Cell Biol. (2012). doi:10.1038/ncb2384

48. Gracz, A. D., Puthoff, B. J. \& Magness, S. T. Identification, Isolation, and Culture of Intestinal Epithelial Stem Cells from Murine Intestine. Methods Mol. Biol. 879, 89-107 (2012).

49. Macosko, E. Z. Z. et al. DropSeq, experimental procedures. Cell (2015). doi:10.1016/j.cell.2015.05.002

50. Buenrostro, J. D., Wu, B., Chang, H. Y. \& Greenleaf, W. J. ATAC-seq: A method for assaying chromatin accessibility genome-wide. Curr. Protoc. Mol. Biol. (2015). doi:10.1002/0471142727.mb2129s109

51. Hatakeyama, M. et al. SUSHI: An exquisite recipe for fully documented, reproducible and reusable NGS data analysis. BMC Bioinformatics (2016). doi:10.1186/s12859-016-1104-8 
52. Robinson, M. D., McCarthy, D. J. \& Smyth, G. K. edgeR: A Bioconductor package for differential expression analysis of digital gene expression data. Bioinformatics (2009).

doi:10.1093/bioinformatics/btp616

53. Kolde, R. Package 'pheatmap'. Bioconductor (2012).

54. Sergushichev, A. A. An algorithm for fast preranked gene set enrichment analysis using cumulative statistic calculation. bioRxiv (2016). doi:10.1101/060012

55. Liberzon, A. et al. The Molecular Signatures Database Hallmark Gene Set Collection. Cell Syst. (2015). doi:10.1016/j.cels.2015.12.004

56. Chen, E. Y. et al. Enrichr: Interactive and collaborative HTML5 gene list enrichment analysis tool. BMC Bioinformatics (2013). doi:10.1186/1471-2105-14-128

57. Kuleshov, M. V. et al. Enrichr: a comprehensive gene set enrichment analysis web server 2016 update. Nucleic Acids Res. (2016). doi:10.1093/nar/gkw377

58. Parekh, S., Ziegenhain, C., Vieth, B., Enard, W. \& Hellmann, I. zUMls - A fast and flexible pipeline to process RNA sequencing data with UMls. GigaScience (2018). doi:10.1093/gigascience/giy059

59. Stuart, T. et al. Comprehensive integration of single cell data. bioRxiv (2018). doi:10.1101/460147

60. Barkas, N. et al. Joint analysis of heterogeneous single-cell RNA-seq dataset collections. Nat. Methods (2019). doi:10.1038/s41592-019-0466-z

61. Angerer, P. et al. Destiny: Diffusion maps for large-scale single-cell data in R. Bioinformatics (2016). doi:10.1093/bioinformatics/btv715

62. Mereu, E. et al. matchSCore: Matching Single-Cell Phenotypes Across Tools and Experiments. bioRxiv (2018). doi:10.1101/314831

63. Kanehisa, M. KEGG: Kyoto Encyclopedia of Genes and Genomes. Nucleic Acids Res. (2000). doi:10.1093/nar/28.1.27

64. Fabregat, A. et al. The Reactome Pathway Knowledgebase. Nucleic Acids Res. (2018). doi:10.1093/nar/gkx1132

65. Ashburner, M. et al. Gene ontology: Tool for the unification of biology. Nature Genetics (2000). doi:10.1038/75556

66. Wickham, H. ggplot2: elegant graphics for data analysis. Journal of the Royal Statistical Society: Series A (Statistics in Society) (2016). doi:10.1007/978-3-319-24277-4

67. Langmead, B. \& Salzberg, S. Bowtie2. Nat. Methods (2013). doi:10.1038/nmeth.1923.Fast

68. Davis, C. A. et al. The Encyclopedia of DNA elements (ENCODE): Data portal update. Nucleic Acids Res. (2018). doi:10.1093/nar/gkx1081

69. Quinlan, A. R. \& Hall, I. M. BEDTools: A flexible suite of utilities for comparing genomic features. Bioinformatics (2010). doi:10.1093/bioinformatics/btq033

70. Liao, Y., Smyth, G. K. \& Shi, W. The R package Rsubread is easier, faster, cheaper and better for alignment and quantification of RNA sequencing reads. Nucleic Acids Res. (2019). doi:10.1093/nar/gkz114

71. Robinson, J. T. et al. Integrative genomics viewer. Nature Biotechnology (2011). doi:10.1038/nbt.1754 


\section{Methods}

\section{Mice}

The Ctnnb1-flox allele ${ }^{44}$ was combined with the Ctnnb1-delC, Ctnnb1-dm or Ctnnb1-D164A7, and bred into the VillinCre-ER ${ }^{\mathrm{T} 2}$ mouse strain (The Jackson Laboratory) to generate villin-

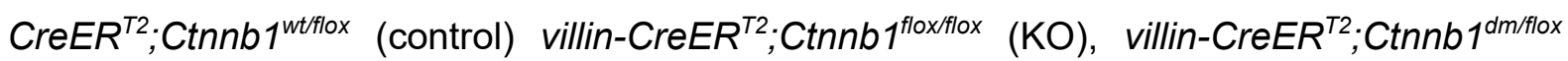

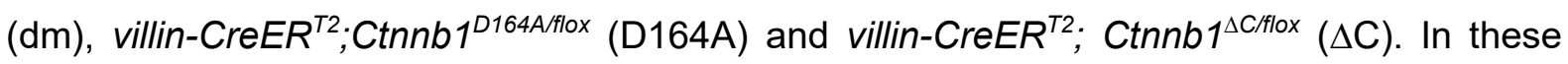
mice, the conditional $\beta$-catenin allele can be deleted specifically in the intestinal epithelium by tamoxifen-inducible VillinCre-ER ${ }^{\mathrm{T} 2}$-mediated recombination, leaving $\mathrm{Wnt} / \beta$-catenin-mediated transcription under the sole control of mutant $\beta$-catenin ( $w$ in control animals). To induce Cre$E R^{\top 2}$-mediated recombination, tamoxifen (Sigma, $80 \mathrm{mg} / \mathrm{kg}$ ) was injected intraperitoneally on two consecutive days. Mice were sacrificed 48 hours after the first tamoxifen injection. Alternatively, mice were injected on 4 consecutive days and sacrificed 96 hours after the first injection. Mouse experiments were performed in accordance with Swiss guidelines and approved by the Veterinarian Office of the Kanton of Zurich, Switzerland. All animals were kept on a C57BL/6 background. Mice were 8-12 weeks old at the time of treatments and cell isolations. Mice of both sexes were used in all experiments and littermates were used as controls.

\section{Duodenal organoids}

Duodenal crypts were isolated and cultured as described ${ }^{45}$ with minor modifications. The duodenum was dissected out, flushed thoroughly, opened longitudinally and then cut in 1-2 $\mathrm{mm}$ stripes. After repeated washing in ice-cold PBS, the duodenal fragments were incubated for 20 min in Gentle Cell Dissociation Reagent (Stemcell Technologies). Crypts were gradually released from the tissue during 4 rounds of washing in $0.2 \%$ BSA and filtering through a 70 micron filter, generating 4 fractions. Only fractions 3 and 4 containing intestinal crypts were used for downstream experiments (organoids or single cell suspension). Crypts were seeded in $50 \mu \mathrm{L}$ Matrigel (Corning) domes and cultured in Intesticult (Stemcell Technologies). Upon passaging, the culture medium was supplemented with $1 \mu \mathrm{M}$ Rho-associated protein kinase (ROCK) inhibitor (Y27632, Millipore). Recombination was induced with 100 nM 4hydroxytamoxifen (4OHT, Sigma) added to the culture medium upon splitting.

\section{METHOD DETAILS}

\section{Mouse genotyping}

The Ctnnb1-D164A allele was detected with the primers 5'-TCCCTGAGACGCTAGATG-3' and 5'-GAGTCCCAGCAGTACAAC-3', yielding an amplicon of the size $475 \mathrm{bp}$ for the wildtype and 628 bp for mutant alleles ${ }^{7}$. Ctnnb1- $\Delta C$ allele was determined using primers 5'- 
GTGCACACGTCATGCTTTAC-3' and 5'-TGGCTTGTCCTCAGACATTCG-3', which generate an amplicon of size $349 \mathrm{bp}$ for the wild-type and $415 \mathrm{bp}$ for mutant alleles ${ }^{7}$. The presence of the villin-CreER ${ }^{T 2}$ allele was detected with the primers 5'-CAAGCCTGGCTCGACGGCC-3' and 5'-CGCGAACATCTTCAGGTTCT-3', which generate a 220 bp product ${ }^{16}$. Recombination of the conditional $\beta$-catenin allele was confirmed for every mouse included in this study by PCR with the primers 5'-AAGGTAGAGTGATGAAAGTTGTT-3' (RM41), 5'CACCATGTCCTCTGTCTATTC-3' (RM42) and 5'-TACACTATTGAATCACAGGGACTT-3' (RM43), generating products of 221 bp for the wild-type allele, 324 bp for the floxed allele and $500 \mathrm{bp}$ for the floxdel allele ${ }^{44}$ ( Extended data Fig. 1d).

\section{Immunohistochemistry}

Dissected duodenum was flushed thoroughly in ice-cold PBS, then cut in $1 \mathrm{~cm}$ pieces and fixed in $4 \%$ PFA in PBS overnight at $4^{\circ} \mathrm{C}$. After repeated washing in PBS, tissues were dehydrated in a spin tissue processor, embedded in paraffin and cut in $8 \mu \mathrm{m}$ sections. Deparaffinized tissue sections were subjected to antigen retrieval in $2.4 \mathrm{mM}$ sodium citrate and $1.6 \mathrm{mM}$ citric acid, $\mathrm{pH}$ 6, for $25 \mathrm{~min}$ in a steamer. Sections were washed with PBST $(0.1 \%$ Tween-20 in PBS) and blocked for $30 \mathrm{~min}$ at RT in blocking buffer (5\% BSA, 5\% heatinactivated normal goat serum in PBST). Following overnight incubation at $4^{\circ} \mathrm{C}$ with primary antibody (1:100, in blocking buffer), sections were washed in PBST and incubated with secondary antibody (1:400, in blocking buffer) for $1 \mathrm{hr}$ at room temperature. Nuclei were stained with DAPI (Sigma, 1:1000) in blocking solution for $5 \mathrm{~min}$ at RT. Sections were imaged on a Leica LSM 710 confocal microscope and processed equally using the ImageJ (FIJI) software, or scanned on Vectra 3.0 Automated Quantitative Pathology Imaging System (Perkin Elmer).

\section{Double histology for intermediate cells}

Deparaffinized tissue sections were incubated with hydrogen peroxide for 5 min to block endogenous peroxidase, then incubated for $30 \mathrm{~min}$ in blocking buffer (5\% BSA, 5\% heatinactivated normal goat serum in PBST) for $30 \mathrm{~min}$. After overnight incubation with primary anti-Lyz antibody (Dako), sections were repeatedly washed in PBST and incubated with biotinylated secondary antibody for $1 \mathrm{~h}$ at $\mathrm{RT}$ and then visualized with VECTASTAIN ABC HRP Kit (Vector Laboratories) as indicated by the manufacturer. Sections were then incubated in a $3 \%$ aqueous solution of acetic acid for $3 \mathrm{~min}$ and then mucins were stained with alcian blue (1 $\mathrm{g}$ in $100 \mathrm{~mL} 3 \%$ acetic acid) for $15 \mathrm{~min}$. Hematoxylin (50\% aqueous solution) and eosin staining were then carried out according to standard procedure. Sections were scanned on Vectra 3.0 Automated Quantitative Pathology Imaging System (Perkin Elmer). 


\section{RNA in situ hybridization}

Duodenum was flushed thoroughly in ice-cold PBS, then cut in $1 \mathrm{~cm}$ pieces and fixed under gentle agitation in $10 \%$ formalin for $20 \mathrm{hrs}$ at room temperature. Axin2 mRNA in situ hybridization was performed with the ACD RNAscope kit (ACDBio), according to the manufacturer's instructions. Sections were image on a Vectra 3.0 Automated Quantitative Pathology Imaging System and quantification was performed with the inForm Cell Analysis software (Perkin Elmer).

\section{Single molecule in situ hybridization}

Mice were sacrificed and the duodenum was removed and flushed thoroughly in ice-cold PBS. Duodenal tissue was then cut in $1 \mathrm{~cm}$ pieces and fixed in 4\% PFA (Santa Cruz Biotechnology, sc-281692) in PBS for 3 hours and subsequently agitated in $30 \%$ sucrose, $4 \%$ PFA in PBS overnight at $4^{\circ} \mathrm{C}$. Fixed tissues were embedded in Tlssue-Tek OCT Compound (Sakura, 4583). $8 \mu \mathrm{m}$ thick sections were sectioned onto poly L-lysine coated coverslips. Probe libraries were designed using the Stellaris FISH Probe Designer Software (Biosearch Technologies) (see Extended data Table 1) and coupled to Cy5 (Myc, Fos, Creb3/3) or TMR (Lgr5, JunB) as described ${ }^{46}$. The intestinal sections were hybridized with smFISH probe sets according to a previously published protocol ${ }^{47}$. DAPI (Sigma-Aldrich) was used as nuclear counterstain. smFISH imaging was performed on a Leica THUNDER 3D Live Cell Imaging system using the following THUNDER Computational Clearing Settings, Feature Scale (nm): 350, Strength (\%): 98, Deconvolution settings: Auto and Optimization: High.

\section{Organoid formation assay and EdU staining}

Mice were sacrificed 0,2 or $4 \mathrm{~d}$ post injection, crypts were isolated as described ${ }^{45}$. Fractions 3 and 4 were seeded in equal amounts (500 crypts) in $50 \mu \mathrm{L}$ Matrigel domes. Organoids were quantified manually 7 day post crypt seeding and micrographs were collected using the AmScope 5.2. software. For EdU staining, crypts were seeded on glass-bottomed 8-well chambered slides (Thermo Fisher Scientific, Lab-TekTM, 154532) in 20 $\mu \mathrm{L}$ drops. EdU incorporation (30 min pulse) and visualization was performed according to the manufacturer instruction with Edu Click 647 Kit (baseclick) prior to overnight incubation with anti-Ecadherin (BS Transduction Lab) antibody for cell shape staining. Imaging was performed on a Visitron CSU-W1 spinning disk confocal microscope with a CFI PlanFluor 20x objective.

\section{RNA sequencing}

Control ( $n=3), K O(n=2), d m(n=3), \Delta C(n=2)$ and D164A ( $n=3)$ mice were sacrificed 2d pi and the duodenal epithelial RNA isolation was performed as described ${ }^{48}$. Briefly, dissected 
duodenum was flushed thoroughly in ice-cold PBS, then opened longitudinally and incubated for 20 min on ice in dissociation buffer 1 (30 mM EDTA, $1.5 \mathrm{mM}$ DTT in PBS), followed by 10 min incubation in dissociation buffer 2 (30 mM EDTA in PBS) at $37^{\circ} \mathrm{C}$ under gentle agitation. The epithelium was released by vortexing, pelleted by centrifugation at $4{ }^{\circ} \mathrm{C}$ for $5 \mathrm{~min}$ at $1000 \mathrm{~g}$, and resuspended in $500 \mu \mathrm{L}$ TRI Reagent (Sigma). RNA was extracted by phenol-chloroform precipitation. DNAse treatment was carried out with DNA-free DNA Removal Kit (Invitrogen) according to the manufacturer's instructions. Library preparation was performed with the Illumina TruSeq RNA Kit. RNA sequencing was performed on the Illumina NextSeq500 by the Functional Genomics Centre Zurich (FGCZ).

\section{Droplet-based single cell mRNA sequencing}

Control $(n=3)$ and D164A $(n=3)$ mice were sacrificed 0,2 or $4 d$ post injection, crypts were isolated as described ${ }^{45}$. Crypt fractions 3 and 4 were pooled and dissociated to single cells by incubating them with $5 \mathrm{~mL}$ pre-warmed TripLE Express Enzyme (ThermoFischer) in a $37^{\circ} \mathrm{C}$ water bath for $5 \mathrm{~min}$ with frequent agitation. TripLE was inactivated with $50 \%$ FBS in Advanced DMEM/F12 (ThermoFischer). Single cells were pelleted at $180 \mathrm{~g}$ for $3 \mathrm{~min}$ and resuspended in $2 \mathrm{~mL}$ cold Intesticult (Stemcell Technologies Inc). Clumps were dissolved by pipetting up and down with a $1 \mathrm{~mL}$ pipet before filtering twice through a 40 micron filter. Cell viability and number were quantified in automatically with a Countess (Thermo Scientific). DropSeq workflow was performed as described ${ }^{49}$ on a Nadia Instrument (Dolomite Bio). Sequencing was performed on the Illumina NovaSeq6000 with an SP Reagent Kit.

\section{ATAC-Seq}

Control $(n=3)$ and D164A $(n=3)$ mice were sacrificed 2d pi and processed independently for ATACSeq library preparation, following the protocol by ${ }^{50}$. Briefly, single cell suspension of duodenal crypts was performed as for scRNAseq, which ensures virtually absent contamination from non-epithelial cells. 50'000 cells were lysed in $50 \mathrm{ml}$ cold Lysis buffer (10 $\mathrm{mM}$ Tris- $\mathrm{HCl}, \mathrm{pH}$ 7.4, $10 \mathrm{mM} \mathrm{NaCl}, 3 \mathrm{mM} \mathrm{MgCl2,} \mathrm{0.1 \%} \mathrm{IGEPAL} \mathrm{CA-630).} \mathrm{After} \mathrm{centrifugation}$ at $500 \mathrm{~g}$ for $10 \mathrm{~min}$ at $4^{\circ} \mathrm{C}$, cells were resuspended in $50 \mathrm{~mL} 1 \mathrm{X}$ Illumina transposition mix (25ml Tagment DNA Buffer, 2.5ml Tagment DNA Enzyme, 22.5ml nuclease-free $\mathrm{H} 2 \mathrm{O}$ ) and incubated for $30 \mathrm{~min}$ at $37^{\circ} \mathrm{C}$ on a shaker. Immediately following the transposition reaction, DNA was purified using the QIAgen MinElute PCR Purification Kit according to the manufacturer's instructions. Library was amplified with Nextera Sequencing primers in NEBNext Hot Start High-Fidelity 2X PCR master mix for 5 cycles. The appropriate number of additional PCR cycles was determined by qPCR. Amplified libraries were purified using the QIAgen MinElute PCR Purification Kit. DNA was eluted in 20ml EB buffer and quantified and 
visualized for quality control with a 2200 TapeStation System (Agilent). Libraries were sequenced on an Illumina HiSeq2500 with paired end 70 bp read configuration.

\section{QUANTIFICATION AND STATISTICAL ANALYSIS}

\section{Quantification of immunofluorescence and RNA in situ}

Percentage of crypt cells positive for Ki67 antibody staining and Axin2+ RNA in situ hybridization were automatically quantified with the software inForm Cell Analysis software (Perkin Elmer). Crypt area was manually defined. We quantified at least 30 crypts from 2-3 different mice per condition. Barplots were generated on GraphPad Prism. The unpaired Student's T test function in GraphPad Prism was used to analyze the significance of two-group comparisons.

\section{smFISH quantification}

The of Lgr5+ area per crypt was manually quantified using FIJI. We quantified at least 8 crypts from 3 different mice per condition. Barplots were generated on GraphPad Prism. The unpaired Student's T test function in GraphPad Prism was used to analyze the significance of two-group comparisons.

\section{Bulk RNA-seq analysis}

Reads were quality-checked with FastQC. Reads alignment to the reference genome "Mus_musculus.GRCm38.95" and read count was performed on the Support Users for SHell script Integration (SUSHI) framework ${ }^{51}$, with the RSEMApp application. Pairwise comparisons were performed with the SUSHI application EdgeRApp (based on edgeR ${ }^{52}$ ). Principal component analysis and filtering of differentially expressed genes (DEGS, logFC > $|2|, p<$ 0.01) were performed on $R$ (version 3.6.1). The package pheatmap ${ }^{53}$ was used to generate the heatmap of normalized FPKM. We performed gene set enrichment analysis ${ }^{18}$ on significantly differentially expressed genes ( $\log F C>|2|, p<0.01)$ using the Bioconductor package fgsea with default parameters ${ }^{54}$. Genes were ranked based on $p$-value, and taking into account directionality of the fold change with the formula ranking $=-\log 10(P) /$ sign(log2ratio) (obtained from the blogpost http://genomespot.blogspot.co.at/2016/04/howto-generate-rank-file-from-gene.html). The Hallmarks gene set collection from the Molecular Signatures Database ${ }^{55}$ was imported in $\mathrm{R}$ with the package msigdbr. Cell type enrichment analysis of DEGs across conditions was performed on the web-based tool EnrichR ${ }^{56,57}$.

\section{Computational analysis of scRNaseq data}

\section{Dimensionality reduction and clustering}


Reads were quality-checked with FastQC. Raw data processing of fastq files was performed with the zUMls pipeline ${ }^{58}$ using the reference genome "Mus_musculus.GRCm38.95". scRNaseq analysis was performed with Seurat 3 on R 3.6.159. Cells were filtered based on mitochondrial gene content, unique molecular identifier (UMI) counts were log-normalized according to default Seurat settings. Scaling was performed on the variable genes (FindVariableFeatures, parameters: x.low.cutoff $=0.0125$, x.high.cutoff $=3$, y.cutoff $=0.5$ ) regressing out UMI number and mitochondrial gene content. Samples were merged and joint analysis was performed with the package $\operatorname{conos}^{60}$. Cells were clustered with the leiden community method (resolution=1.3). The joint graph (in PCA space) was embedded in UMAP space and converted to a Seurat object. The stem cell and early progenitor clusters were subsetted and the log-normalized UMI counts were exported and used for the normalization algorithm and downstream analysis.

\section{Normalization algorithm}

Analysis of the control timecourse data revealed that the acute loss of one $\beta$-catenin allele only caused a slight and transient downregulation of Wnt-targets, proliferation and stem markers, which then returned to normal expression levels by $4 \mathrm{~d}$ pi. Thus, we devised a normalization of the mutant timecourse data to disentangle these effects from those induced by impaired N-terminal interactions, which were the focus of our investigation. For every timepoint (0, 2 and 4d pi), we divided the D164A single-cell expression (from data slot) of each gene by the mean expression of the corresponding gene in the control with the formula: $\ln [\exp (D 164 A) /($ mean $(\exp ($ control $)]$. The resulting three normalized D164A sparse matrices (one per timepoint) were used to create Seurat objects, which were merged, scaled and visualized in UMAP space using dimensions 1:10.

\section{Diffusion maps and pseudotime analysis}

For diffusion maps and supervised pseudotime analysis, the normalized Seurat object was converted in a SingleCellExperiment. Diffusion maps were generated with the Bioconductor package destiny ${ }^{61}$ using default parameters. Scores in the diffusion component 1 were compared using the Wilcoxon test (alternative: two.sided) in R base. Supervised pseudotime analysis was performed with the Bioconductor package psupertime ${ }^{26}$ on all genes or on mouse transcription factors only, setting scale to FALSE.

\section{Cell cycle scoring and classification}

Cell cycle scoring was performed with the CellCycleScoring algorithm from Seurat, using cell cycle-related genes from ${ }^{25}$. Wilcoxon test (alternative: two.sided) was used to compare G2M scores. To assign a measure of similarity to IESCs or TAs transcriptomic profiles, we trained 
a logistic regression (modification of the multinomial regression of MatchSCore $2^{62}$ using IESC and TA markers extracted from a publicly available single cell dataset ${ }^{29}$. Upon collecting the estimated probabilities of TA class memberships for each timepoint separately, we compared these probability distributions and tested the significance of the difference using KolmogorovSmirnov test (alternative: two.sided).

\section{Gene set enrichment analysis}

We used the Seurat FindMarkers function (default parameters min.pct 0.25 and logfc.threshold 0.25 ) to compute differentially expressed genes between timepoints in our normalized D164A stem cell and early progenitor dataset. We then ranked genes with the formula ranking $=-\log 10\left(p_{-} v a l_{-} a d j\right) / \operatorname{sign}\left(a v g \_l o g F C\right)$ and performed gene set enrichment analysis $^{18,55}$ on $\mathrm{R}$ using the Bioconductor package fgsea with default parameters ${ }^{54}$. The following gene set collections were imported in $\mathrm{R}$ with the package msigdbr: Hallmarks ${ }^{55}$, Chemical and Genetic Perturbations (various contributions), KEGG ${ }^{63},{ }^{64}$ and gene ontology biological process ${ }^{65}$. Plots were generated with the R package ggplot $2^{66}$.

\section{Computational ATAC-Seq analysis}

Reads were quality-checked with FastQC. Adapters trimming with cutadapt, alignment on GRCh38.95 using Bowtie2 with default mapping parameters ${ }^{67}$, as well as duplicate filtering with Picard and peak calling with MACS2 $(p<0.01)$ were performed on the ENCODE pipeline ${ }^{68}$ supported by the SUSHI application AtacENCODEApp, for every biological replicate separately. The peak files were merged in BEDtools v2.29.2 ${ }^{69}$ and converted into a saf annotation, to which raw reads were mapped with the FeatureCounts function in the package rsubread $^{70}$. The resulting count matrix was filtered for peaks with low coverage (minimum read count of 10 for each sample). The remaining counts were then normalized by total library size and TMM-derived normapllization factors calculated with edge ${ }^{52}$. Differential peaks between sample groups were identified using the exact test function in edgeR. Only peaks with a log fold change $>1$ and an $p$ value $<0.01$ were considered as differentially accessible for further analysis. HOMER ${ }^{36}$ was used for peak annotation and motif enrichment analysis. Motifs with Benjamini-corrected q-value $<0.001$ were considered significantly enriched. bigWig files of control $(n=3)$ and D164A $(n=3)$ were combined into a single track for visualization in Integrative Genomics Viewer ${ }^{71}$. Genes associated with a differentially accessible peak were ranked with the formula ranking $=-\log 10($ Pvalue $) / \operatorname{sign}(\log 2 F C)$ and used for gene set enrichment analysis with the $\mathrm{R}$ using the Bioconductor package fgsea with default parameters ${ }^{54}$. Significance of overlap between gene sets overrepresented in scRNAseq and in ATACSeq was quantified with hypergeometric test. Plots were generated with the R package ggplot $2^{66}$. 


\section{DATA AND CODE AVAILABILITY}

Data generated in this study are deposited in GEO with the accession numbers: GSE148941, GSE148942, GSE148940. The code used in this study are available at the repository https://github.com/cocoborrelli/betacat. 


\section{Figure Legends}

Figure 1. Attenuation of $\mathrm{N}$ - vs. C-terminal $\beta$-catenin transcriptional outputs has contrasting effects on intestinal homeostasis. A) Scheme of wt and mutant $\beta$-catenin proteins with impaired interactions to $\mathrm{N}$ - or C-terminal transcriptional co-factors. B) Principal component analysis (PCA) of transcriptome of mutant and control intestinal epithelium $2 \mathrm{~d}$ pi. PC1 and PC2 explain $94.8 \%$ and $3 \%$ of the variance, respectively. C) Upset plot of intersecting Differentially Expressed Genes (DEGs) (logFC > $2 \mid, p<0.01)$ in $\Delta C$ (C-terminal mutant), dm (double mutant), KO (complete loss), D164A (N-terminal mutant), with respect to control. D) Heatmap of normalized FPKM of Wnt and IESC marker genes across hierarchically clustered mutants. Rows are scaled. E) Axin2 (Wnt-target) mRNA in situ hybridization, Olfm4 (IESC marker) and Ki67 (proliferating cells) immunofluorescence of control, D164A and $\triangle \mathrm{C}$ duodenal crypts 2d pi. Hematoxylin, DAPI (nuclei) or E-cadherin (cell membrane) as counterstain. Arrows indicate Ki67+ cells at crypt bottom. F) Quantification of Axin2+ and Ki67+ cells per crypt. G-H) smFISH of $L g r 5$ and Myc mRNA. Arrows indicate single mRNA molecules visible as dots. Red dashed line indicates Lgr5+ stem cell compartment. Scale bars, $20 \mu M$. Student's T-test, unpaired, ${ }^{* *} p<0.01,{ }^{*} p<0.05$. Abbreviations: KO: villin-CreER ${ }^{T 2}$;Ctnnb ${ }^{\text {floxfflox }}$, dm: villinCreER ${ }^{T 2}$;Ctnnb1 $1^{\text {dm/flox }} \quad$ control: villin-CreER ${ }^{T 2} ; C_{\text {Cnnb1 }}{ }^{\text {wtflox }}$, D164A: villinCreER ${ }^{T 2} ;$ Ctnnb1 $^{\text {D164A/flox }}, \Delta \mathrm{C}:$ villin-CreER ${ }^{T 2} ;$ Ctnnb $^{\text {AC/flox }}$

Figure 2. Hyperproliferative intestinal crypts lacking the output via of $\mathrm{N}$-terminal recruited co-factors exhibit stem cell loss and secretory hyperplasia 4d pi. A) Axin2 (Wnt-target) in situ hybridization, Olfm4 (IESC marker), Ki67 (proliferating cells), lysozyme (Lyz, Paneth cells) and Sox9 (Paneth cells per-cursors) immunofluorescence of control and D164A crypts 4d pi. Hematoxylin, DAPI (nuclei) or E-cadherin (cell shape) for counterstain. B) Lyz-HRP (Paneth cells) and alcian blue-stained mucing (goblet cells) double staining. Hematoxylin-eosin as counterstain. C) Working model summarizing impacts of missing contribution of $\mathrm{N}$ - vs. C-terminal $\beta$-catenin co-factors.

Figure 3. Hyperproliferative IESCs expressing only Ctnnb1 ${ }^{D 164 A}$ have an increased ability to form organoids. A) Experimental workflow of organoid formation assay. B) Organoid formation efficiency (in \%) of control (villin-CreER ${ }^{T 2}$;Ctnnb $1^{\text {wtfllox }}$ ) and mutant (villinCreER ${ }^{T 2} ; C_{\text {tnnb1 }}{ }^{\text {D164A/flox }}$ ) crypts isolated 0, 2 and $4 \mathrm{~d}$ pi. C) Brightfield images of control and mutant organoids formed from crypts isolated $2 \mathrm{~d}$ pi. Inset at day $7 \mathrm{EdU}+$ shows EdU staining (proliferating cells). E-cadherin (cell shape) and DAPI (nuclei) as counterstain. Scale bars, 20 $\mu \mathrm{M}$. Student's T-test, unpaired, ${ }^{* * * *} p<0.0001,{ }^{* *} p<0.01,{ }^{*} p<0.05{ }^{*}$.

Figure 4. Myc-E2F-driven proliferation is followed by stress signalling and loss of stem cells in N-terminal mutant (D164A) crypts. A) Diffusion map for normalized D164A stem cells and early progenitors (SCEPs). Cells (points) colored by timepoint after Cre induction (days pi). Diffusion component 1 (DC1) represents differentiation pseudotime. On the right, cells colored by their average expression of stem cell (top) and differentiation markers (bottom). B) Distribution of DC1 score in D164A SCEP cells over time $\left({ }^{* * *} p<0.001\right.$, Wilcoxon test). C) Distribution of cell cycle score (average expression of cycling genes) in SCEPs over 
time $\left({ }^{* * *} p<0.001\right.$, Wilcoxon test). C) Expression profile of stem cell and proliferation markers in SCEPs ordered along supervised pseudotime (psupertime). Cells colored by timepoint. D) Gene set expression analysis GSEA on time-varying genes in SCEPs across timepoints. Normalized enrichment score (y-axis) over time (x-axis) of MSigDB gene sets. Dot size indicates size of enriched gene set. Dot color indicates $p$ value of enrichment. Selected gene sets labeled (identifiers in text). E) CD45 immunofluorescence in control and mutant 4d pi. Details show immune cell (circle) infiltration within mutant crypts (dashed line). Scale bars, 20 $\mu \mathrm{M}$.

Figure 5. JNK signalling mediates major chromatin remodelling and triggers the expression of villus tip genes in D164A crypts. A-B) Venn diagram and volcano plot of ATACseq peaks in control and D164A crypts. Differential peak analysis indicates that 600 peaks are gained $(\log F C>1 \& p<0.01)$ and 869 are lost $(\log F C<-1 \& p<0.01)$ in D164A crypts $2 \mathrm{~d}$ pi. Selected peaks labeled. C) 10 most significantly enriched transcription factor (TF) binding motifs from HOMER motif analysis on differential peaks. D) Combined ATACSeq tracks of control and D164A counts. Black arrows show gained peaks with AP-1 binding motif annotated to Mapk8/JNK and Mapk13/SAP1. E) Immunostaining of phospho-JNK. E-cadherin (cell shape) and DAPI (nuclei) counterstain. F) smFISH of Fos and JunB mRNA. Arrows indicate single mRNA molecules visible as dots. Scale bars, $20 \mu \mathrm{M}$.

Figure 6. Scheme summarizing the distnct roles of $\beta$-catenin transcriptional outputs on the identity of the intestinal epithelial stem cells C-terminal co-activators govern basal Wnt signaling and preventing their activity leads to loss of stem cells and proliferative arrest. Contrary, N-terminal co-factors act as stem cell specifyers. Blocking their contribution results in hyperproliferation and aberrant differentiation of intestinal epithelial stem cells. (IESCintestinal epithelial stem cell, TA-transit amplifier cell) 
Figure 1
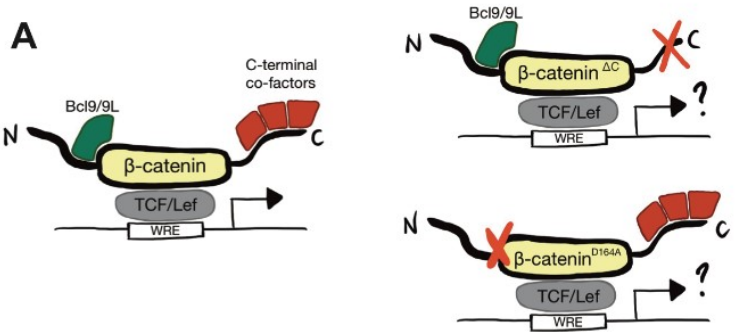

C

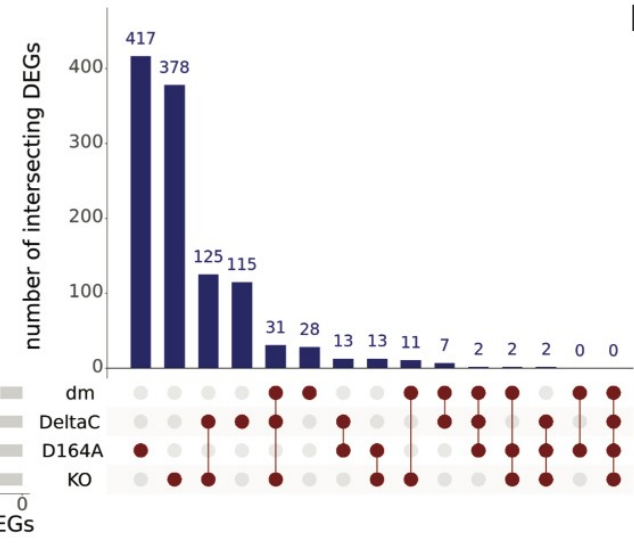

B

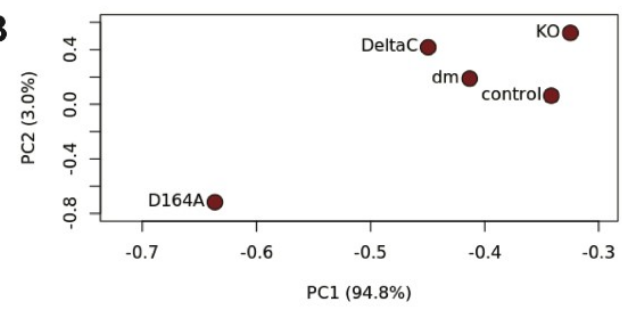

D

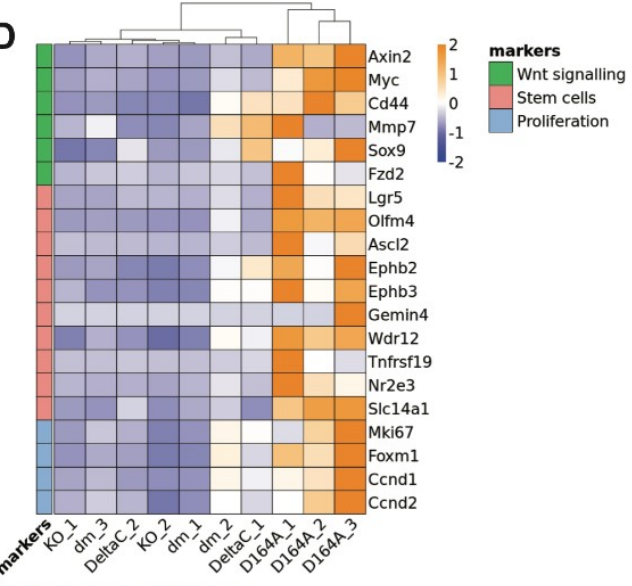

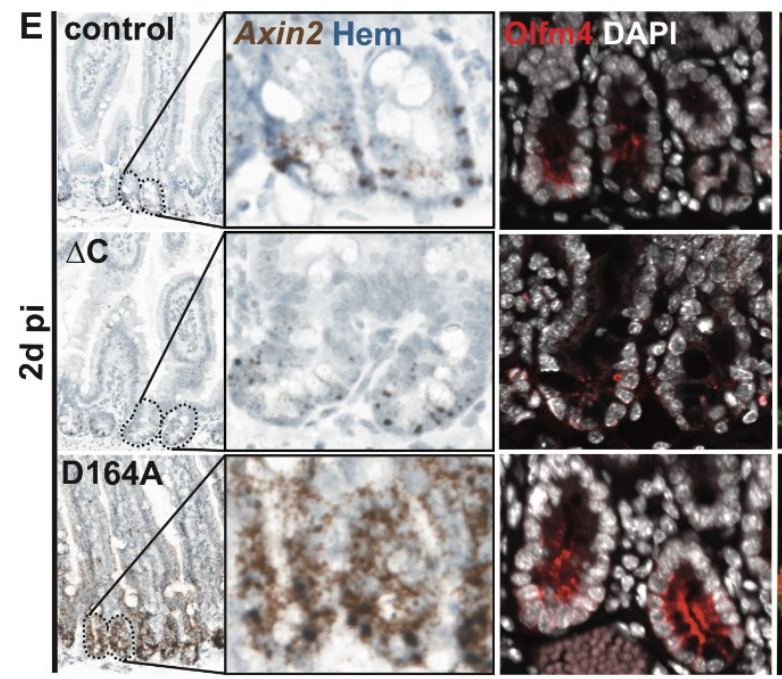

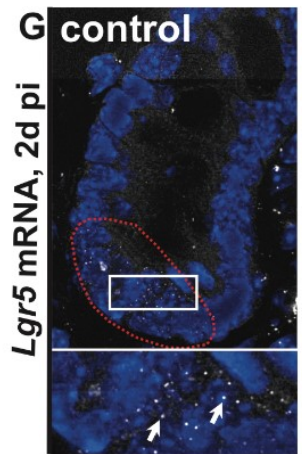

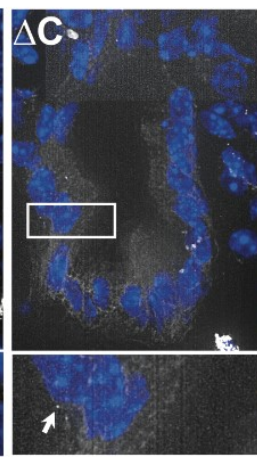

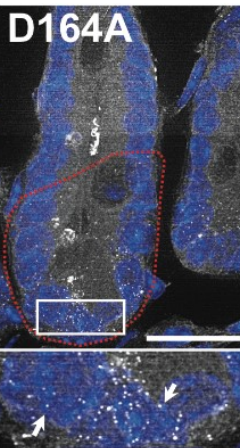

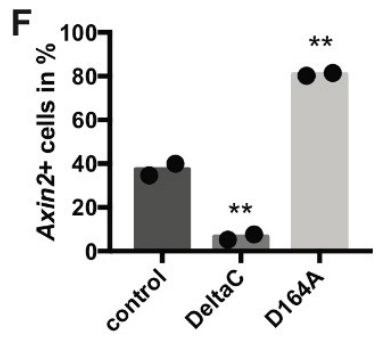
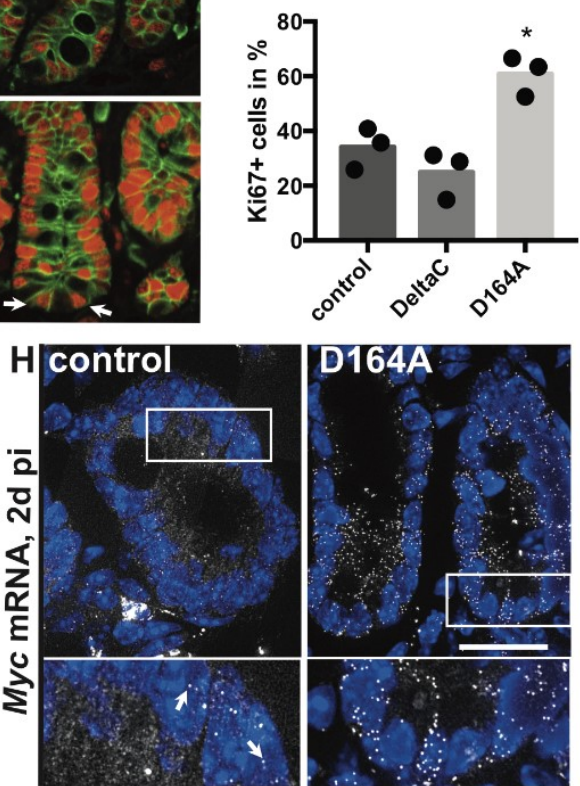
bioRxiv preprint doi: https://doi org/10.1101/2020.05.19.103499. this version posted May 20, 2020. The copyright holder for this preprint (which was not certified by peer review) is the author/funder. All rights reserved. No reuse allowed without permission.

Figure 2
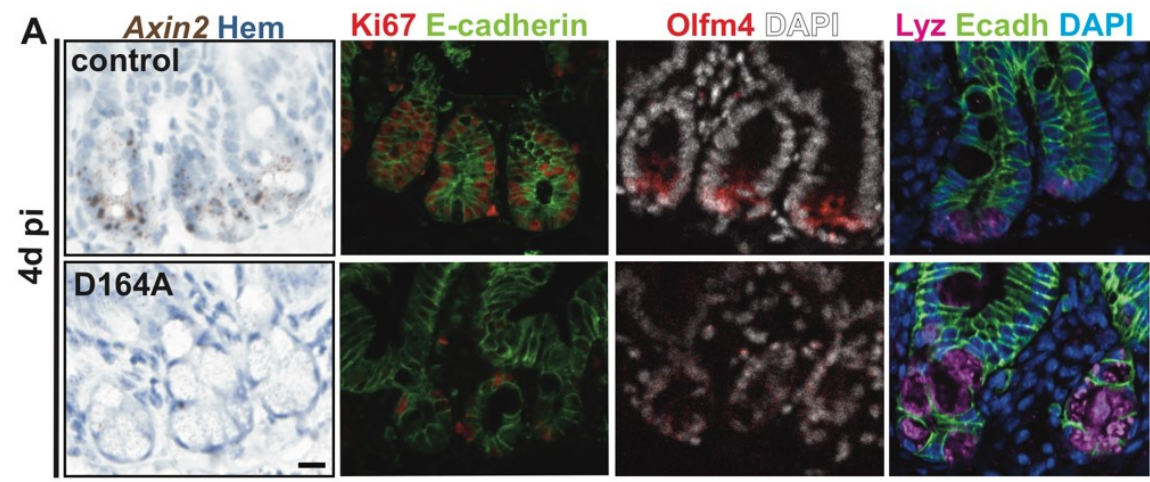

Sox9 DAPI

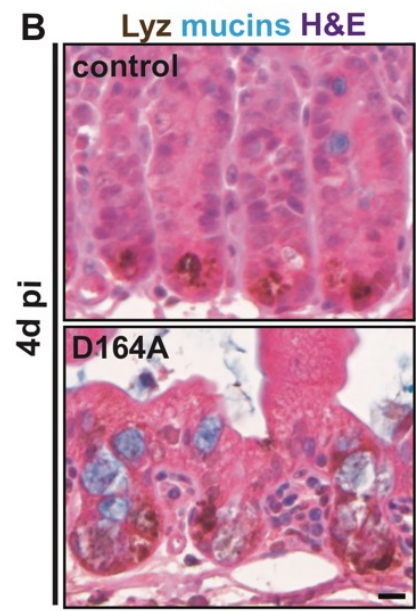

C

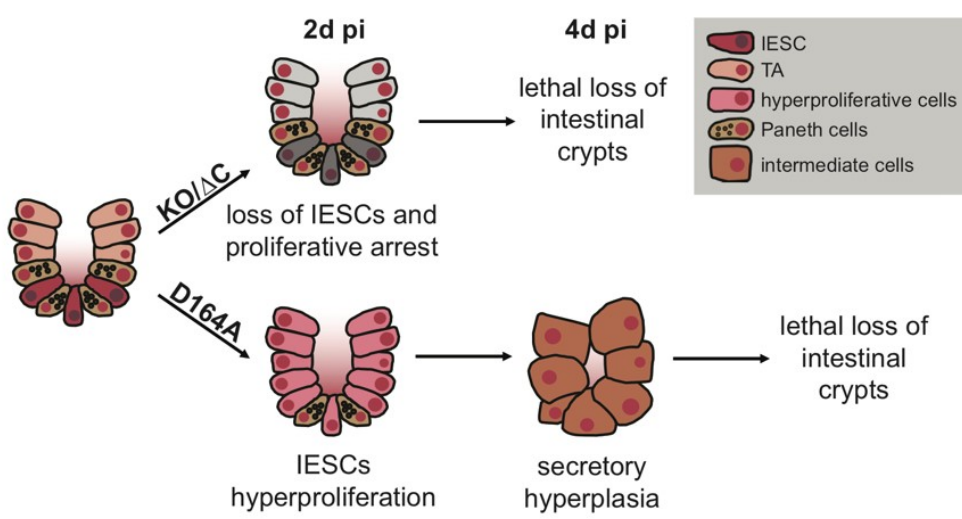


Figure 3

A<smiles>CC1C=C1</smiles>

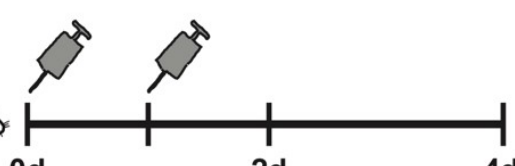

Od 2d
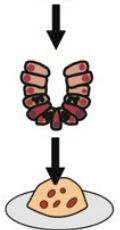

$\downarrow$

8

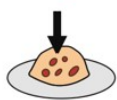

B

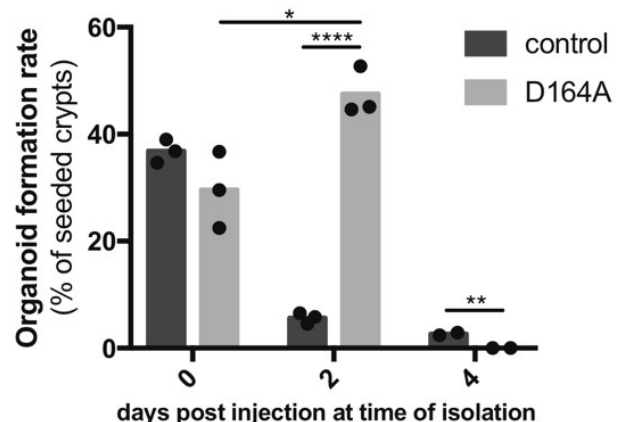

C

day 0

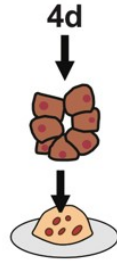

day 3

day 7

post splitting

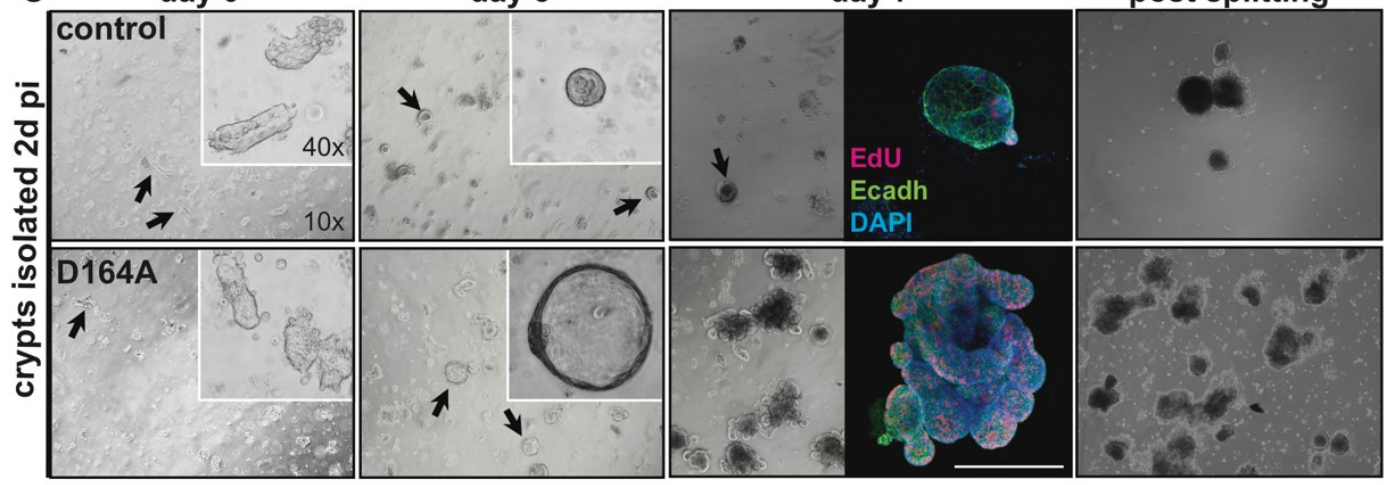


Figure 4
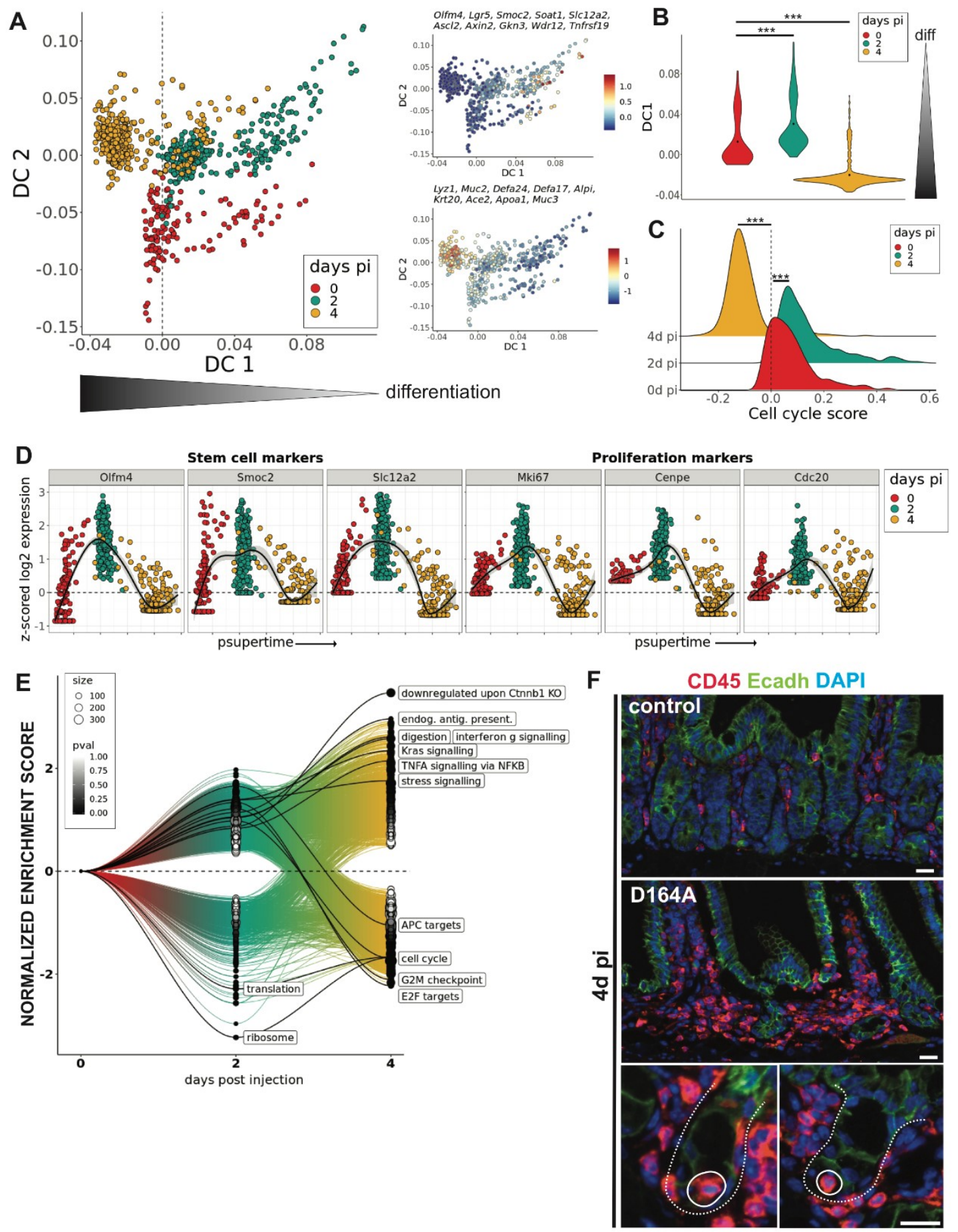
Figure 5

A

B

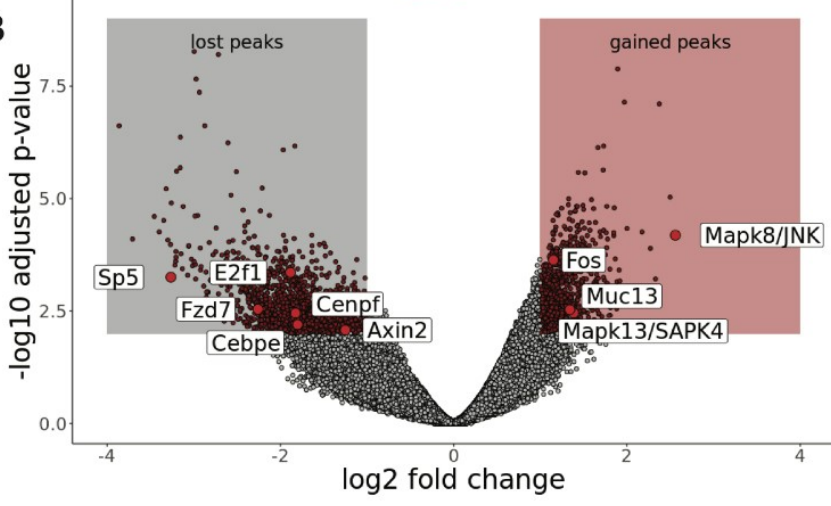

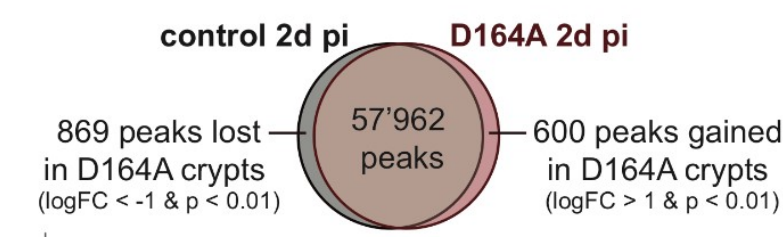

\begin{tabular}{|c|c|c|}
\hline moti & TF & $\mathrm{pV}$ \\
\hline GATCAAAGTCCA & BATF & $1 \mathrm{e}-57$ \\
\hline 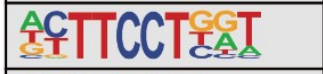 & Junb & $1 \mathrm{e}-57$ \\
\hline $\begin{array}{l}\text { GTCATAAA } \\
\text { EICAT }\end{array}$ & Fra2 & $1 e-$ \\
\hline \begin{tabular}{|l} 
ATGACTCA \\
CTC \\
\end{tabular} & AP-1 & $1 \mathrm{e}-55$ \\
\hline GGTATAAATGAA & Fra1 & $1 e-4$ \\
\hline 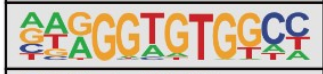 & Atf3 & $1 \mathrm{e}-$ \\
\hline CECTTATC & Fos & $1 \mathrm{e}$ \\
\hline 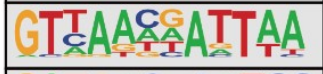 & Fosl2 & $1 \mathrm{e}-$ \\
\hline $\begin{array}{l}\text { GATGACTCATCC } \\
\text { 遂ETGG } \\
\end{array}$ & Jun-AP1 & $1 \mathrm{e}-$ \\
\hline 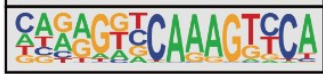 & HNF4a & $1 \mathrm{e}-29$ \\
\hline
\end{tabular}

\section{D}
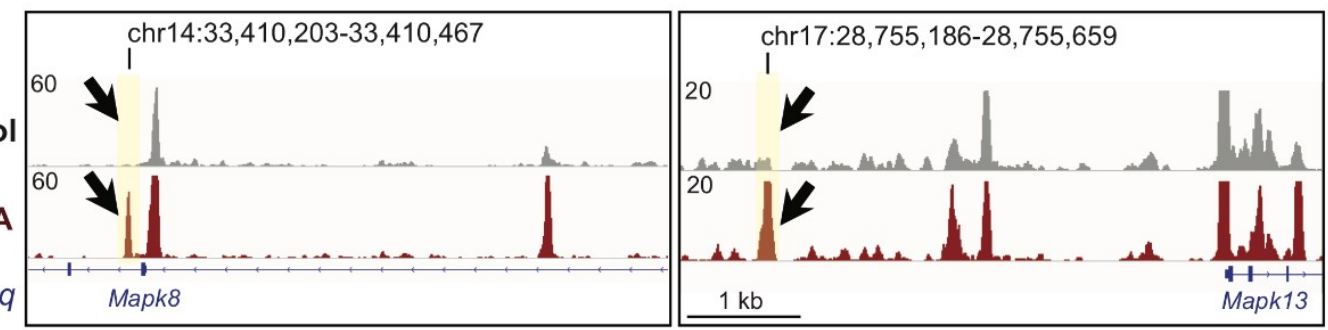

F

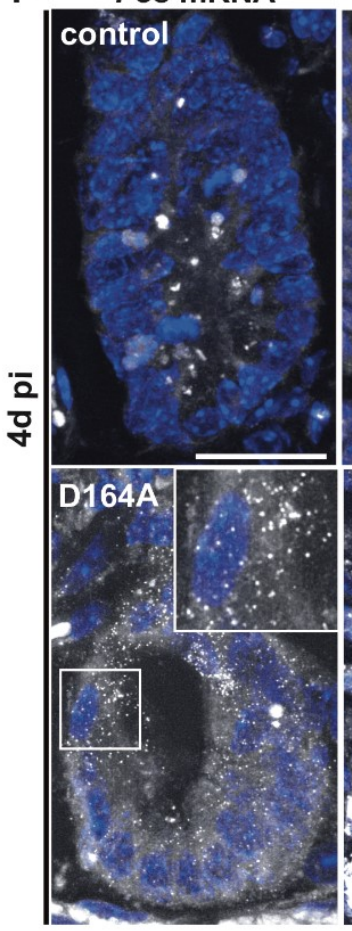

JunB mRNA

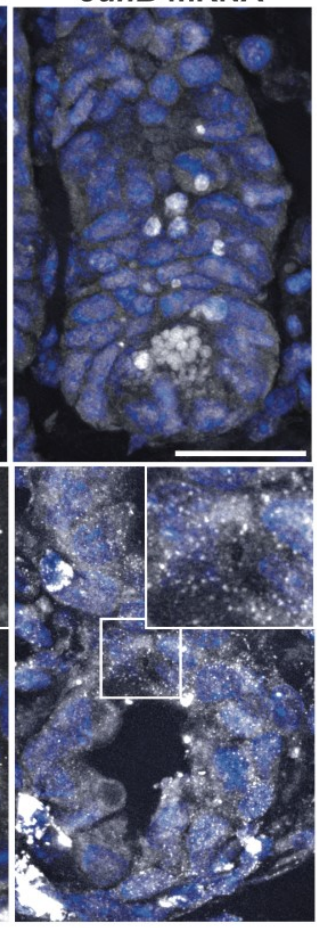


Figure 6

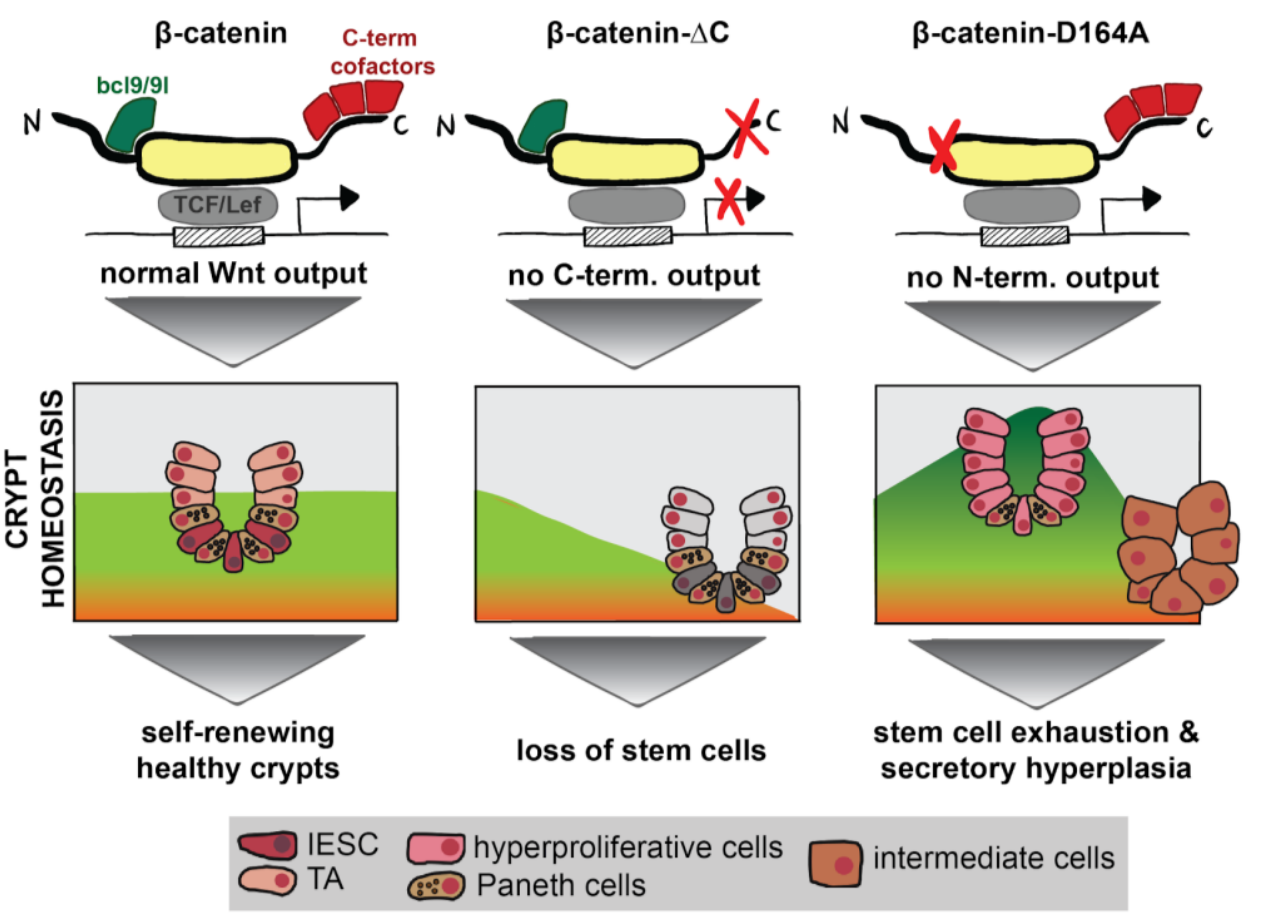




\section{Extended Data}

\section{Extended Data Figure Legend}

Extended Data Figure 1. Distinct effects of $\mathrm{N}$ - vs. C-terminal $\beta$-catenin transcriptional outputs on intestinal homeostasis. A) Scheme of wt, mutant and conditional Ctnnb1 alleles used in this study. B) $\Delta \mathrm{C}$ animals suffer from crypt atrophy and reach humane endpoint $4 \mathrm{~d}$ pi. C) D164A (N-terminal mutant) animals show loss of crypts and villus shortening $7 \mathrm{~d}$ pi. Thickening of the mesenchyme indicates immune infiltration and severe colitis. D) Map of the $\beta$-catenin locus, modified from ${ }^{44}$ depicting how the recombination of conditional allele was confirmed. Red triangles indicate the loxP sequences flanking exons 2 (which contains the ATG) to 6. Prior to recombination, primers RM41 and RM42 generate a 221 bp band for the wt allele and a $324 \mathrm{bp}$ band for the floxed allele, respectively. Upon Cre induction, primers RM42 and RM43 generate a 500 bp band. Representative PCR showing successful and tissue-specific recombination in DNA obtained from the intestinal epithelium but not from the tail. E) Depletion of wt (flox) $\beta$-catenin in the crypts of $\Delta \mathrm{C}$ animals $2 \mathrm{~d} p \mathrm{p}$, as shown by immunofluorescence with antibody specific for $\beta$-catenin's C-terminal moiety. F) Gene set enrichment analysis (GSEA) on differentially expressed genes (DEGs, logFC > $2 \mid, p<0.05$ ) of $\mathrm{KO}, \mathrm{dm}$ and $\triangle \mathrm{C}$ animals. Annotated gene sets obtained from the Hallmarks collection of the Molecular Signatures Database (MSigDB). G) Cell type enrichment analysis of DEGs across mutants performed on the web-based tool EnrichR ${ }^{56,57}$. H) Percentage of Lgr5+ (IESC marker) area per crypt in control and D164A animals. Student's T-test, unpaired, * $p<0.05$. Scale bars, $20 \mu \mathrm{M}$.

Extended Data Figure 2. $\beta$-catenin is haploinsufficient in vitro. A) villinCreER ${ }^{T 2} ;$ Ctnnb $^{\text {wtfllox }}$ and villin-CreER ${ }^{T 2} ;$ Ctnnb1 $^{\text {D164A/flox }}$ organoids treated with 4hydroxytamoxifen (4OHT) in vitro die 7 days after recombination. Duodenal organoids lacking the villin-CreER ${ }^{T 2}$ allele are not affected by $4 \mathrm{OHT}$. Days after addition of $4 \mathrm{OHT}$ indicated at the top. B) Duodenal organoids derived from Ctnnb $1^{\triangle C \text { /flox }}$ and Ctnnb $1^{\text {dm/flox }}$, as well as from constitutively hemizygous Ctnnb $1^{K O / w t}$ animals mice grow slower than wt controls and can not be propagated in vitro. 20x magnification.

Extended Data Figure 3. Longitudinal scRNASeq of control and $\mathrm{N}$-terminal mutant (D164A) crypts. A) UMAP embedding of joint graph of single cells sequenced from control and D164A crypts isolated 0,2 and $4 \mathrm{~d}$ pi. Leiden clustering results in 10 clusters corresponding to the main cell populations in the intestinal crypt. B) Marker gene expression across manually annotated clusters. C) Cluster breakdown in percent across all samples. D) Heatmap of the top 200 differentially regulated genes in normalized D164A stem cells and early progenitors (SCEP) cells across timepoints. IESC and proliferation markers and upregulated $2 \mathrm{~d}$ pi, while expression of differentiation markers increases $4 \mathrm{~d}$ pi. E) Distribution of predicted model responses of logistic regression showing a shift of SCEPs $2 \mathrm{~d}$ pi towards TA traits. Kolmogorov-Smirnov test $\left({ }^{* * *} \mathrm{p}<0.001\right)$. F) Supervised pseudotime ordering coefficients of mouse transcription factors in decreasing absolute value. G) smFISH of Creb3/3 mRNA evidences its ectopic expression in D164A crypts 4d pi. Red and green dotted lines indicate crypt and villus area, respectively. Scale bars, $20 \mu \mathrm{M}$. 
Extended data Figure 4. ATAC sequencing of control and N-terminal mutant (D164A) crypts. A) Results of HOMER motif enrichment on 1469 differential ATAC peaks (logFC > $|1|$ $\& p<0.01$ ) between control and N-terminal mutant (D164A) crypts. Number of target sequences on $x$-axis, - $\log 10$ ( $p$-value) of enrichment on y-axis. Significantly enriched motifs (Benjamini-corrected q-value < 0.001) depicted in red and labeled with corresponding transcription factors. B) Venn diagrams and upset plot showing overlaps between enriched pathways (logFC $>|1|$ ) in N-terminal mutant (D164A) crypts, compared to controls, as revealed by ATACSeq ( $2 \mathrm{~d}$ pi) and scRNASeq ( $4 \mathrm{~d}$ pi). Significance of the overlap of overrepresented pathways calculated with hypergeometric test $\left({ }^{* *} p<0.001\right)$. 

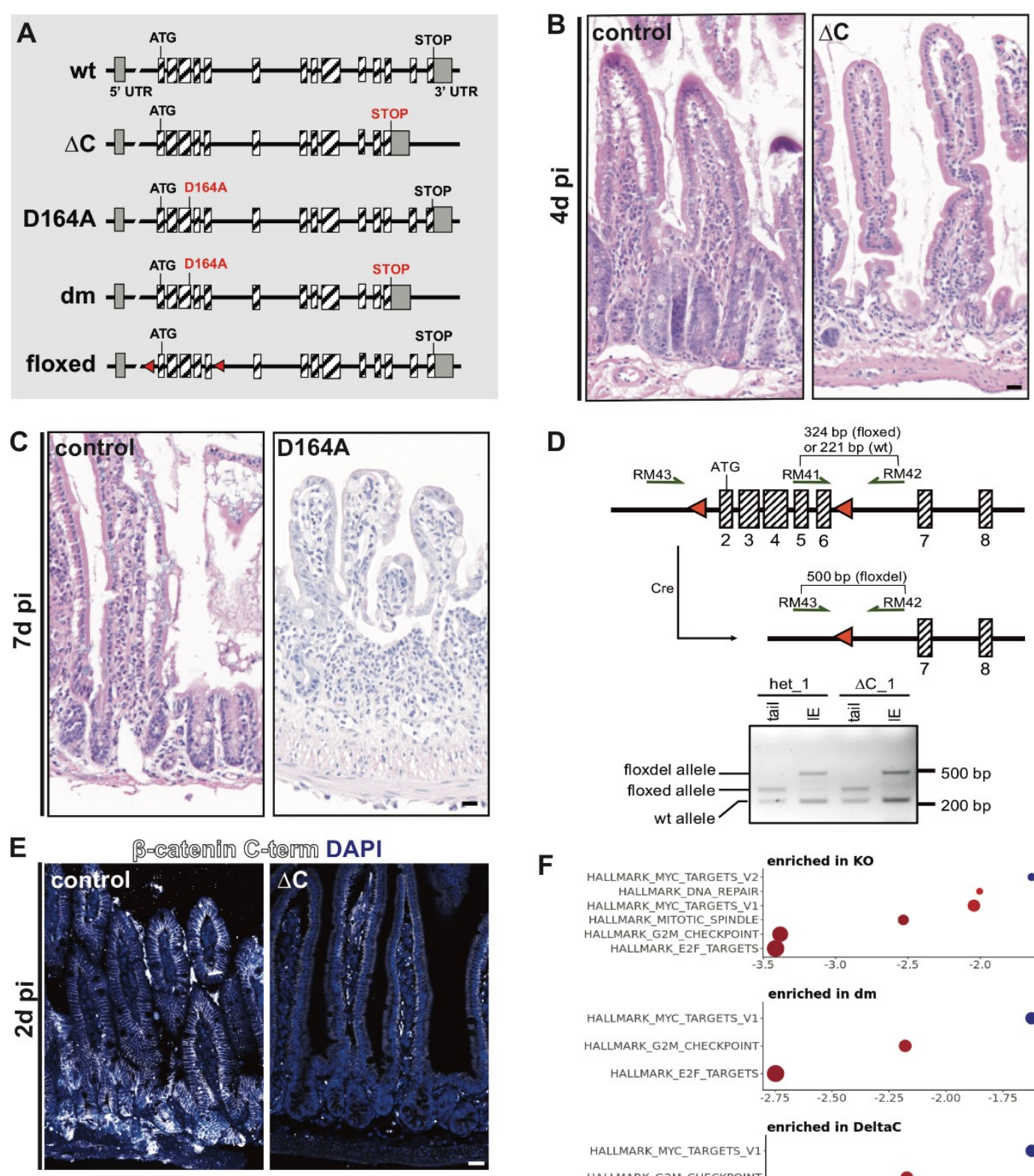

G enrichR cell type enrichment (Mouse Cell Atlas)

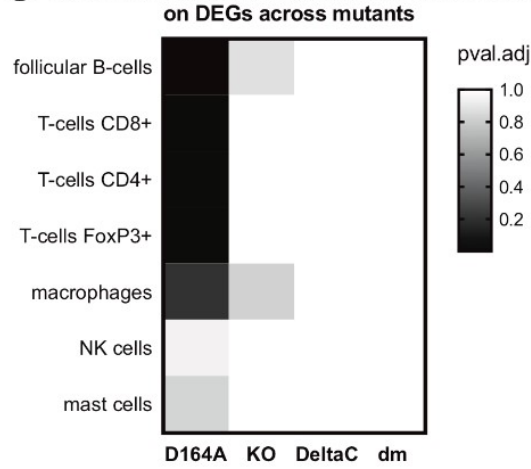

F

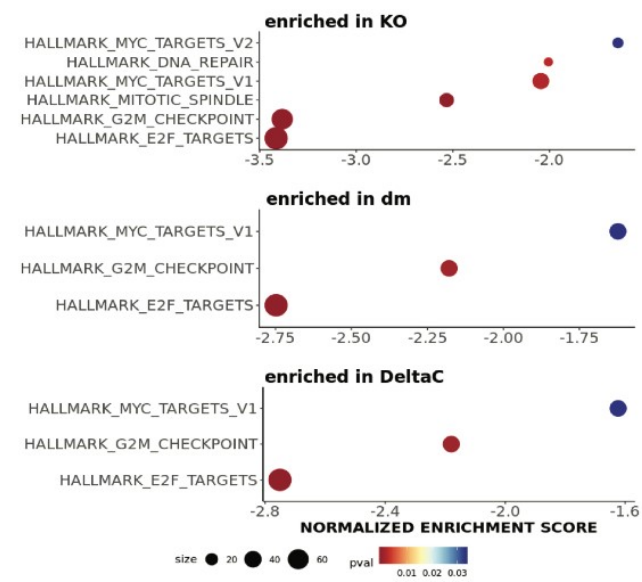

H

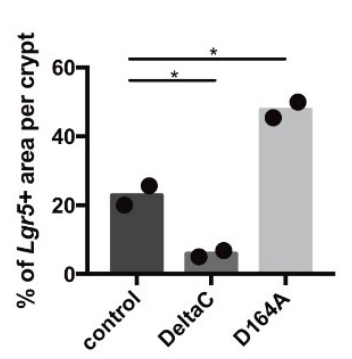




\section{A 40HT}
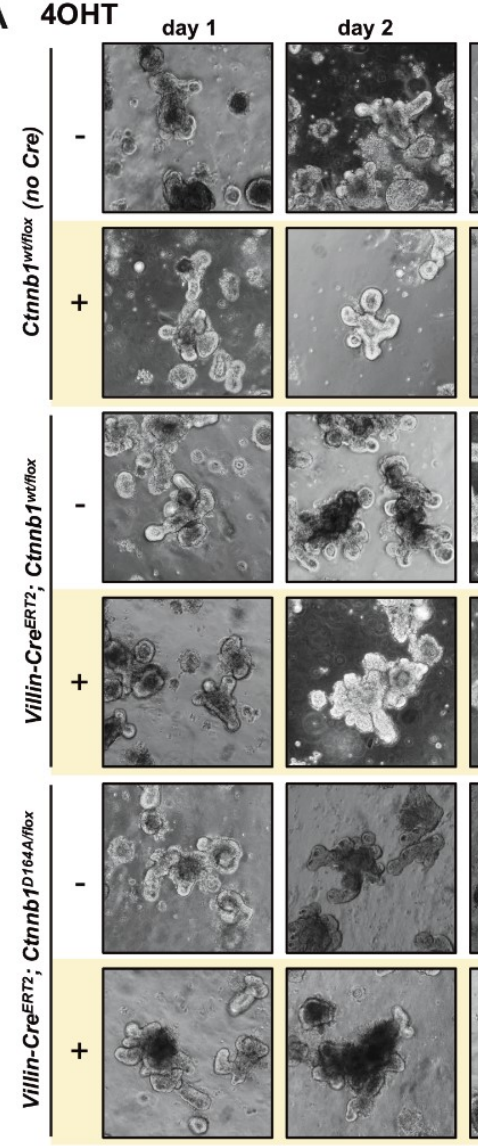
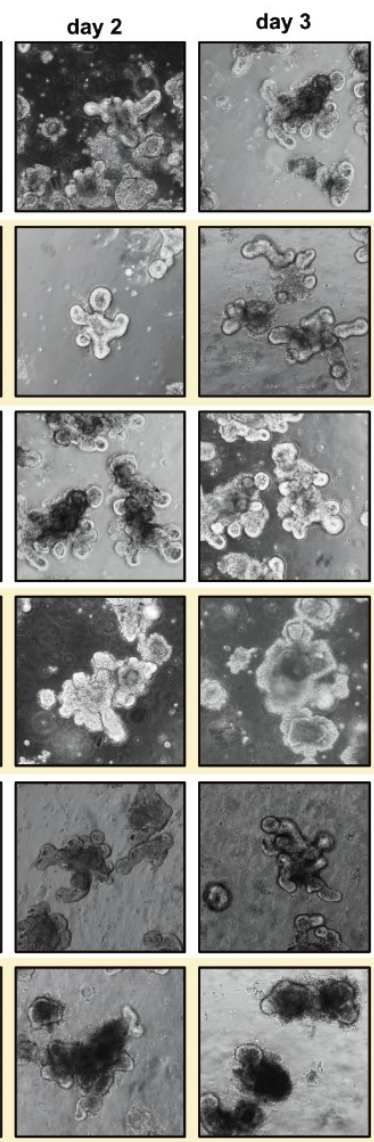
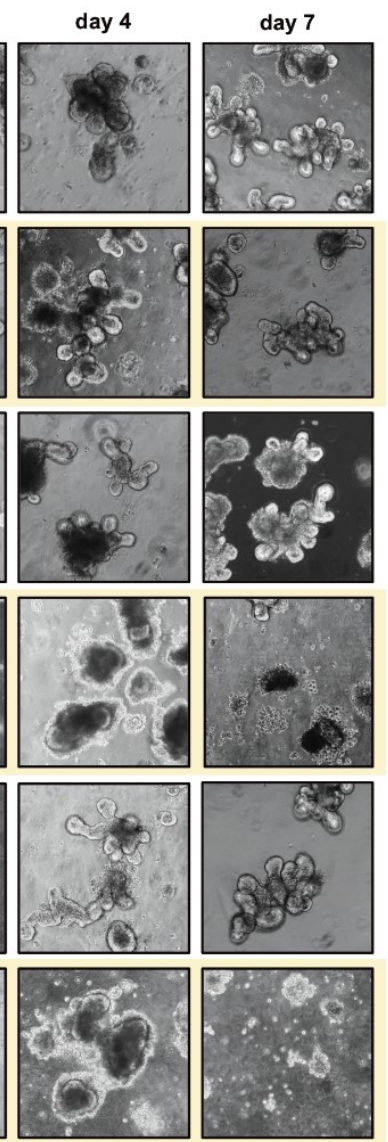

B
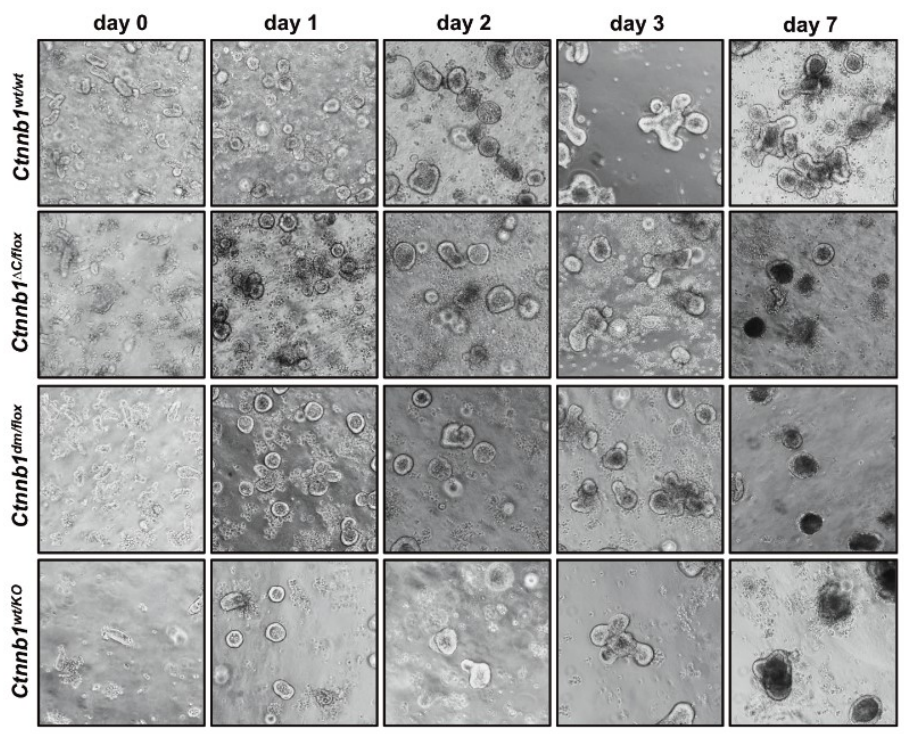
bioRxiv preprint doi: https://doi.org/10.1101/2020.05.19.103499. this version posted May 20, 2020. The copyright holder for this preprint (which was not certified by peer review) is the author/funder. All rights reserved. No reuse allowed without permission.

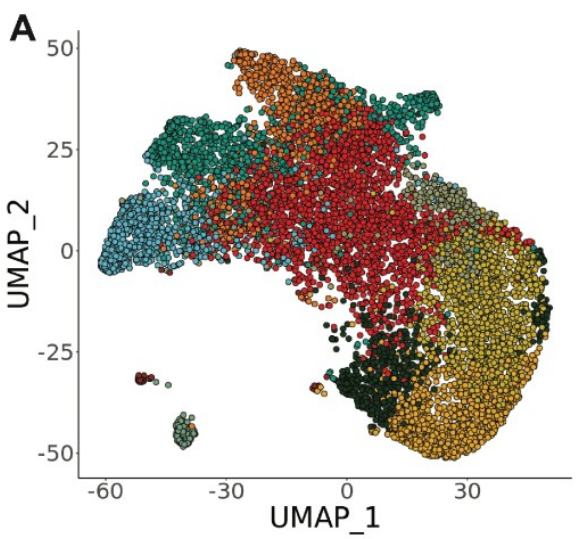

\section{Cluster breakdown per sample}
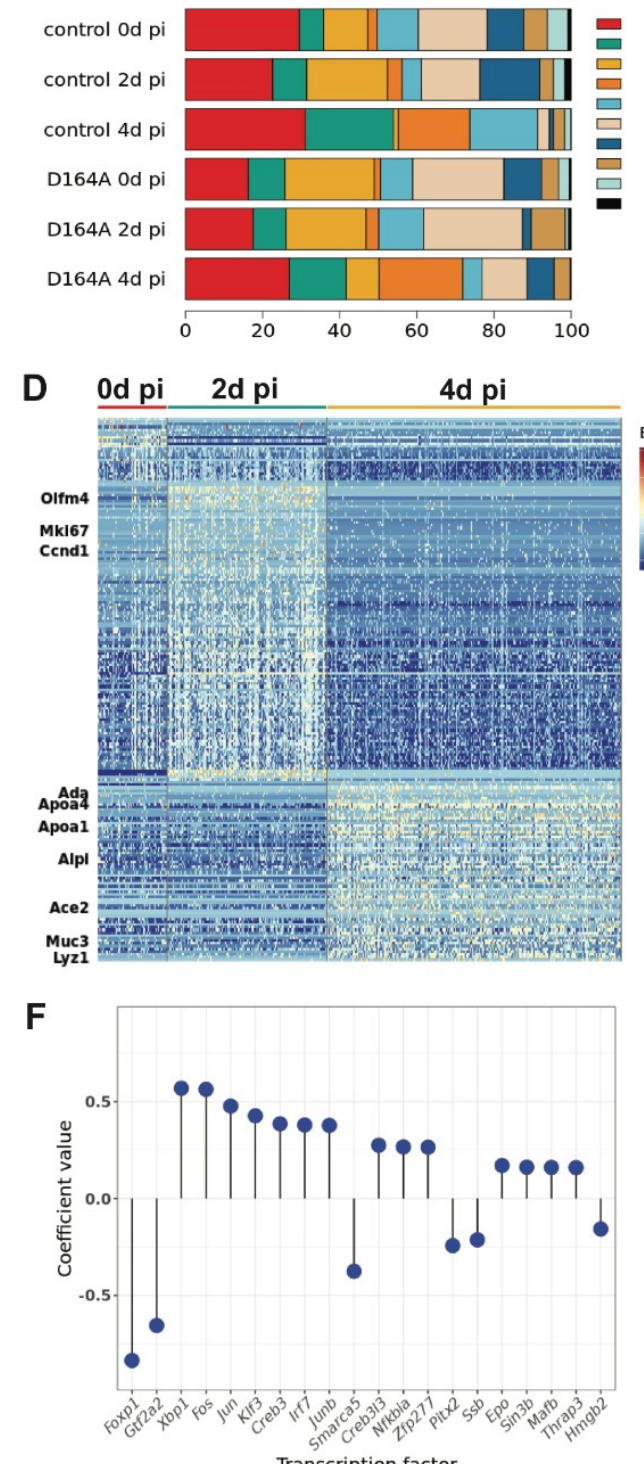

CLUSTER

- Early progenitors

Goblet cells

Paneth cells

Stem cells 2

Enterocytes (immature)

T cells

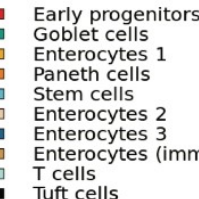

Early progenitors

Goblet cells

Paneth cells

tem cells

Enterocytes

Theres

cells

Tuft cells

B

B Average Expression Percent Expressed

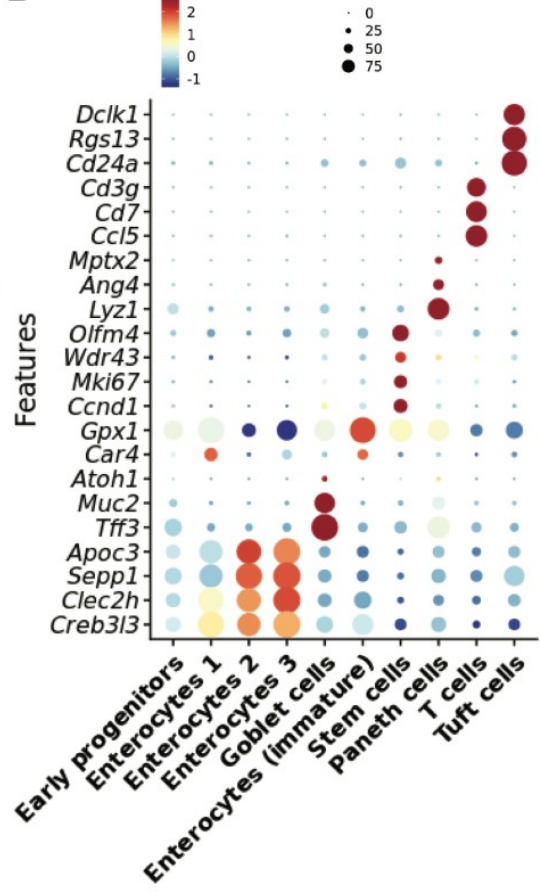

E

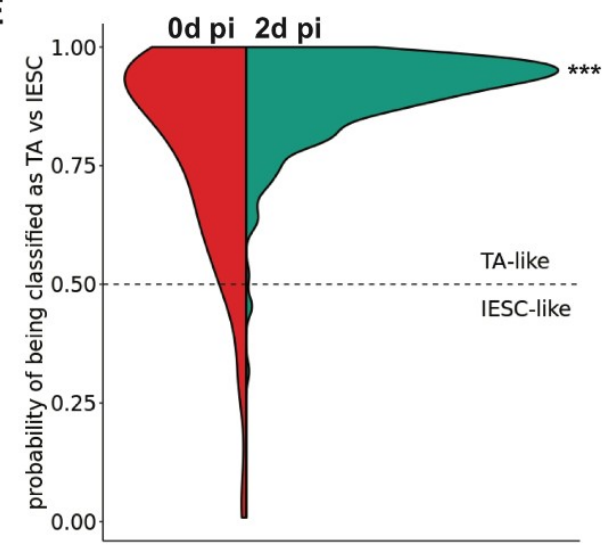

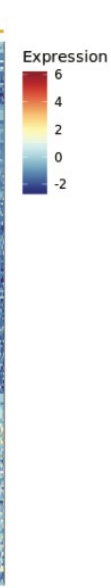
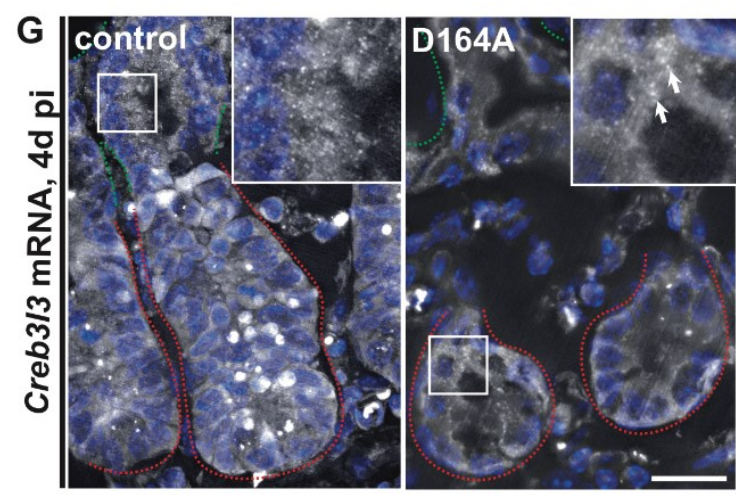
bioRxiv preprint doi: https://doi.org/10.1101/2020.05.19.103499. this version posted May 20, 2020. The copyright holder for this preprint (which was not certified by peer review) is the author/funder. All rights reserved. No reuse allowed without permission.

\section{Extended Data Figure 4}

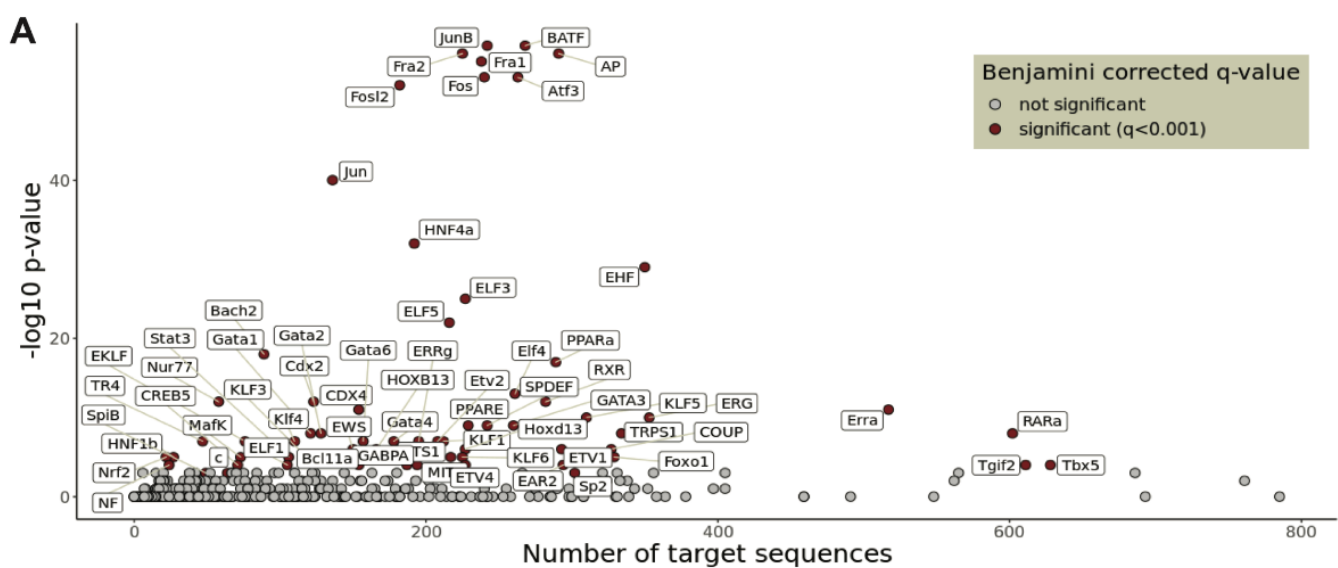

B
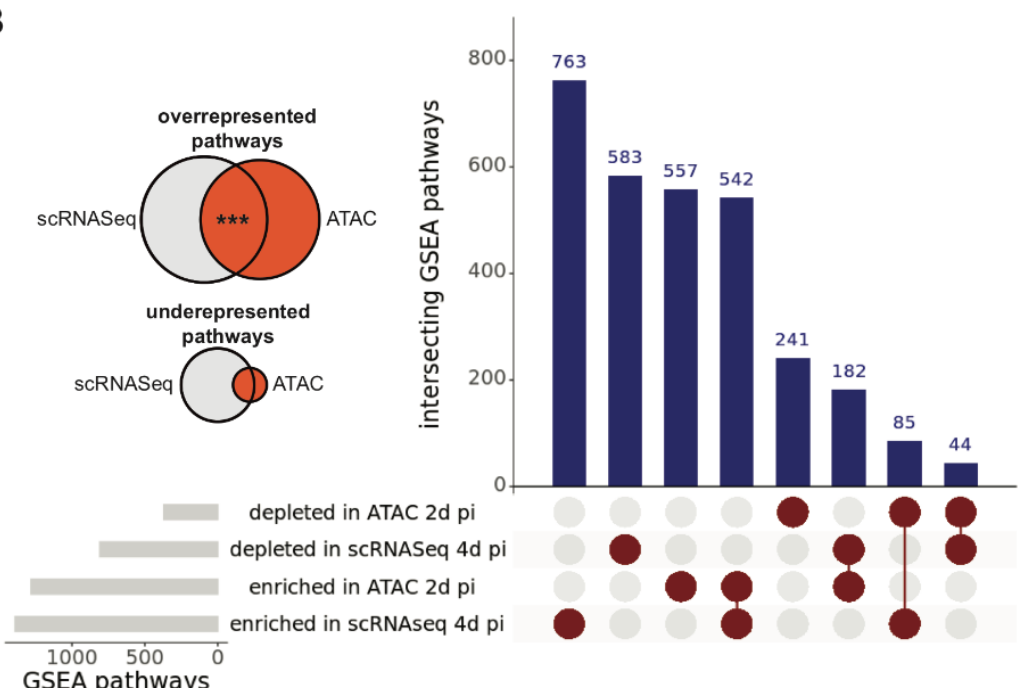

GSEA pathways 


\section{Extended Data Methods}

\section{KEY RESOURCES TABLE}

\begin{tabular}{|c|c|c|}
\hline REAGENT or RESOURCE & SOURCE & IDENTIFIER \\
\hline \multicolumn{3}{|l|}{ Antibodies } \\
\hline mouse monoclonal anti $\beta$-catenin (C-terminus) & BD Transduction Lab & $\begin{array}{l}\text { 610153; RRID: } \\
\text { AB_397554 }\end{array}$ \\
\hline rabbit polyclonal anti Ki67 & Abcam & $\begin{array}{l}\text { ab15580; RRID: } \\
\text { AB_443209 }\end{array}$ \\
\hline mouse monoclonal anti E-cadherin & BD Transduction Lab & $\begin{array}{l}\text { 610181; RRID: } \\
\text { AB_397580 }\end{array}$ \\
\hline rabbit polyclonal anti Lysozyme & Dako & $\begin{array}{l}\text { A0099; RRID: } \\
\text { AB_2341230 }\end{array}$ \\
\hline rat monoclonal anti CD45-FITC & Invitrogen & $\begin{array}{l}\text { 12-0453-82; RRID: } \\
A B \_465675\end{array}$ \\
\hline rabbit Sox9 & Sigma Aldrich & $\begin{array}{l}\text { AB5535; RRID: } \\
\text { AB_2239761 }\end{array}$ \\
\hline rabbit monoclonal anti-Olfm4 & Cell Signaling & $\begin{array}{l}\text { 39141; RRID: } \\
\text { AB_2650511 }\end{array}$ \\
\hline rabbit polyclonal anti-ACTIVE ${ }^{\circledR} \mathrm{JNK} \mathrm{pAb}$ & Promega & $\begin{array}{l}\text { V7931; RRID: } \\
\text { AB_430864 }\end{array}$ \\
\hline Alexa fluor 594 goat anti-rabbit & ThermoFischer & $\begin{array}{l}\text { A-11037; RRID: } \\
\text { AB_2534095 }\end{array}$ \\
\hline Alexa fluor 647 goat anti-mouse & ThermoFischer & $\begin{array}{l}\text { A-21236; RRID: } \\
\text { AB_2535805 }\end{array}$ \\
\hline Alexa fluor 594 goat anti-rat & ThermoFischer & $\begin{array}{l}\text { A-11007; RRID: } \\
\text { AB_10561522 }\end{array}$ \\
\hline Peroxidase-AffiniPure Goat Anti-Rabbit IgG & \begin{tabular}{|l} 
Jackson \\
ImmunoResearch
\end{tabular} & $\begin{array}{l}\text { 111-035-144; RRID: } \\
A B \_2307391\end{array}$ \\
\hline \multicolumn{3}{|l|}{ Chemicals } \\
\hline Tamoxifen & Sigma & T5648 \\
\hline Matrigel & Corning & 354230 \\
\hline 4-hydroxytamoxifen & Sigma & $\underline{H 6278}$ \\
\hline ROCK inhibitor Y-27632 & Millipore & SCM075 \\
\hline Alcian Blue & Sigma & A3157 \\
\hline Hematoxylin & Sigma & 51275 \\
\hline Eosin Y-solution & Sigma & HT110280 \\
\hline DAPI & Sigma & D9543 \\
\hline $4 \%$ paraformaldehyde in PBS & \begin{tabular}{|l} 
Santa Cruz \\
Biotechnology
\end{tabular} & sc-281692 \\
\hline TripLE Express Enzyme 1X & ThermoFischer & 12604013 \\
\hline Advanced DMEM/F12 & ThermoFischer & 12634010 \\
\hline Tissue-Tek OCT Compound & Sakura & 4583 \\
\hline Bovine Serum Albumin & Sigma & A9647 \\
\hline Gentle Cell Dissociation Reagent & Stemcell Technologies & 07174 \\
\hline Instesticult Organoid Growth Medium (Mouse) & Stemcell Technologies & 06005 \\
\hline \multicolumn{3}{|l|}{ Critical Commercial Assay } \\
\hline VECTASTAIN ABC HRP Kit & Vector Laboratories & PK-4000 \\
\hline RNAScope 2.5 HD Reagent Kit Brown & ACDBio & 322300 \\
\hline MinElute PCR Purification Kit & Qiagen & 28004 \\
\hline Edu Click 647 Kit + EdU & baseclick GmbH & BCK647-IV-IM \\
\hline
\end{tabular}




\begin{tabular}{|c|c|c|}
\hline \multicolumn{3}{|l|}{ Deposited Data } \\
\hline bulk RNA sequencing & This study & GEO: GSE148941 \\
\hline single cell mRNA sequencing & This study & GEO: GSE148942 \\
\hline bulk ATAC sequencing & This study & GEO: GSE148940 \\
\hline \multicolumn{3}{|l|}{ Experimental Models: Duodenal Organoid Lines } \\
\hline Murine VillinCre-ER ${ }^{\mathrm{T} 2}$-Ctnnb1 164A/flox & This study & $\mathrm{N} / \mathrm{A}$ \\
\hline Murine VillinCre-ER ${ }^{\top 2}$-Ctnnb1 ${ }^{\text {wtfllox }}$ & This study & $\mathrm{N} / \mathrm{A}$ \\
\hline Murine Ctnnb1wt/wt & This study & $\mathrm{N} / \mathrm{A}$ \\
\hline Murine Ctnnb1 ${ }^{\text {wt/KO }}$ & This study & $\mathrm{N} / \mathrm{A}$ \\
\hline Murine Ctnnb1 $1 \mathrm{dm} /$ flox & This study & $\mathrm{N} / \mathrm{A}$ \\
\hline Murine Ctnnb1 $1^{\Delta \mathrm{C} / \mathrm{flox}}$ & This study & $\mathrm{N} / \mathrm{A}$ \\
\hline \multicolumn{3}{|l|}{ Experimental Models: Organisms/Strains } \\
\hline Mus musculus_Ctnnb1-D164A & Valenta et al, 2011 & RRID:MGI: 5308942 \\
\hline Mus musculus_Ctnnb1-delC & Valenta et al, 2011 & RRID:MGI: 5308945 \\
\hline Mus musculus_Ctnnb1-dm & Valenta et al, 2011 & RRID:MGI: 5308947 \\
\hline Mus musculus_Ctnnb1-flox & Brault et al, 2001 & RRID:MGI: 2148567 \\
\hline Mus musculus_Tg(Vil1-cre/ERT2) & $\begin{array}{l}\text { The Jackson } \\
\text { Laboratory }\end{array}$ & RRID:MGI: 3053826 \\
\hline \multicolumn{3}{|l|}{ Oligonucleotides } \\
\hline Fwd primer Ctnnb1-D164A: TCCCTGAGACGCTAGATG & Valenta et al, 2001 & $\mathrm{~N} / \mathrm{A}$ \\
\hline Rev primer Ctnnb1-D164A: GAGTCCCAGCAGTACAAC & Valenta et al, 2001 & N/A \\
\hline Fwd primer Ctnnb1-delC: GTGCACACGTCATGCTTTAC & Valenta et al, 2001 & $\mathrm{~N} / \mathrm{A}$ \\
\hline Rev primer Ctnnb1-delC: TGGCTTGTCCTCAGACATTCG & Valenta et al, 2001 & N/A \\
\hline Fwd primer vilCreER ${ }^{\top 2}$ : CAAGCCTGGCTCGACGGCC & El Marjou et al., 2004 & $\mathrm{~N} / \mathrm{A}$ \\
\hline Rev primer vilCreER ${ }^{\top 2}$ : CGCGAACATCTTCAGGTTCT & El Marjou et al., 2004 & N/A \\
\hline Primer RM41 Ctnnb1-flox: AAGGTAGAGTGATGAAAGTTGTT & Brault et al. 2001 & N/A \\
\hline Primer RM42 Ctnnb1-flox: CACCATGTCCTCTGTCTATTC & Brault et al. 2001 & $\mathrm{~N} / \mathrm{A}$ \\
\hline \begin{tabular}{|l} 
Primer RM43 Ctnnb1-flox: \\
TACACTATTGAATCACAGGGACTT \\
\end{tabular} & Brault et al. 2001 & $\mathrm{~N} / \mathrm{A}$ \\
\hline See Table S1 for smFISH probes & This study & $\mathrm{N} / \mathrm{A}$ \\
\hline \multicolumn{3}{|l|}{ Software and Algorithms } \\
\hline GraphPad Prism v7.0a & $\begin{array}{l}\text { GraphPad Software } \\
\text { Schneider }\end{array}$ & $\begin{array}{l}\text { https://www.graphpad.c } \\
\text { om/scientific- } \\
\text { software/prism/ }\end{array}$ \\
\hline IGV 2.8.0 & Broad Institute & $\begin{array}{l}\text { https://software.broadin } \\
\text { stitute.org/software/igv/ }\end{array}$ \\
\hline R software 3.6 .1 & GNU project & https://www.r-project.org \\
\hline R Studio & RStudio & https://www.rstudio.com \\
\hline SUSHI: Supporting User for SHell script Integration & $\begin{array}{l}\text { Functional Genomics } \\
\text { Center Zurich }\end{array}$ & $\begin{array}{l}\text { https://github.com/uzh/s } \\
\text { ushi }\end{array}$ \\
\hline edgeR R package & Robinson et al, 2010 & $\begin{array}{l}\text { https://bioconductor.org/ } \\
\text { packages/release/bioc/h } \\
\text { tml/edgeR.html }\end{array}$ \\
\hline pheatmap R package & Kolde, 2012 & $\begin{array}{l}\text { https://cran.r- } \\
\text { project.org/web/packag } \\
\text { es/pheatmap/pheatmap. } \\
\text { pdf }\end{array}$ \\
\hline
\end{tabular}




\begin{tabular}{|c|c|c|}
\hline Seurat v3.0 & Stuart et al, 2019 & $\begin{array}{l}\text { https://satijalab.org/seur } \\
\text { at/get_started.html } \\
\text { RRID:SCR_016341 }\end{array}$ \\
\hline conos R package & Barkas et al, 2019 & $\begin{array}{l}\text { https://github.com/hms- } \\
\text { dbmi/conos }\end{array}$ \\
\hline psupertime R package & $\begin{array}{l}\text { Mcnair \& Claassen, } \\
2018\end{array}$ & $\begin{array}{l}\text { https://github.com/wmac } \\
\text { nair/psupertime }\end{array}$ \\
\hline destiny R package & Angerer et al, 2015 & $\begin{array}{l}\text { http://bioconductor.org/p } \\
\text { ackages/release/bioc/ht } \\
\text { ml/destiny.html }\end{array}$ \\
\hline msigdbr R package & R Bioconductor & $\begin{array}{l}\text { https://cran.r- } \\
\text { project.org/web/packag } \\
\text { es/msigdbr/vignettes/ms } \\
\text { igdbr-intro.html }\end{array}$ \\
\hline fgsea R package & $\begin{array}{l}\text { Sergushichev et al, } \\
2016\end{array}$ & $\begin{array}{l}\text { https://bioconductor.org/ } \\
\text { packages/release/bioc/h } \\
\text { tml/fgsea.html }\end{array}$ \\
\hline ggplot2 R package & Wickham, 2016 & $\begin{array}{l}\text { https://cloud.r- } \\
\text { project.org/web/packag } \\
\text { es/ggplot2/index.html }\end{array}$ \\
\hline bedtools & Quinlan and Hall, 2010 & $\begin{array}{l}\text { https://bedtools.readthe } \\
\text { docs.io/en/latest/ }\end{array}$ \\
\hline HOMER v4.11 & Heinz et al, 2010 & $\begin{array}{l}\text { http://homer.ucsd.edu/h } \\
\text { omer/ }\end{array}$ \\
\hline LAS-X & Leica Microsystems & $\begin{array}{l}\text { https://www.leica- } \\
\text { microsystems.com/prod } \\
\text { ucts/microscope- } \\
\text { software/ }\end{array}$ \\
\hline Image J Fiji & Schindelin et al, 2012 & https://imagej.net/Fiji/ \\
\hline inForm Cell Analysis & Perkin Elmer & $\begin{array}{l}\text { https://www.perkinelmer } \\
\text {.com/lab- } \\
\text { solutions/resources/doc } \\
\text { s/BRO_010576_01_PR } \\
\text { D_inForm.pdf }\end{array}$ \\
\hline
\end{tabular}

